\title{
STOCHASTIC PARTIAL DIFFERENTIAL EQUATIONS WITH DIRICHLET WHITE-NOISE BOUNDARY CONDITIONS
}

\author{
Elisa ALÒS ${ }^{\mathrm{a}}$, Stefano BONACCORSI ${ }^{\mathrm{b}}$ \\ a Departament d'Economia i Empresa, Universitat Pompeau Fabra, C/Ramós Trias Fargas, 25-27, \\ 08005 Barcelona, Spain \\ ${ }^{\mathrm{b}}$ Dipartimento di Matematica, Università di Trento, 38050 Povo (Trento), Italy
}

Received 10 November 1999, revised 11 July 2001

ABSTRACT. - The paper is devoted to one-dimensional nonlinear stochastic partial differential equations of parabolic type with non homogeneous Dirichlet boundary conditions of white-noise type. We formulate a set of conditions that a random field must satisfy to solve the equation. We show that a unique solution exists and that we can write it in terms of the stochastic kernel related to the problem. This formulation allows us to study the basic properties of the solution, as the continuity and the boundary-layer behavior, by means of Malliavin calculus. @ 2002 Éditions scientifiques et médicales Elsevier SAS

AMS classification: $60 \mathrm{H} 15 ; 60 \mathrm{H} 07$

RÉSUMÉ. - Cet article est consacré à l'étude d'équations aux dérivées partielles stochastiques non linéaires paraboliques en dimension un avec conditions aux bord de type Dirichlet non homogènes. Nous formulons des conditions qu'un champ aléatoire doit satisfaire pour resoudre l'EDPS. Nous montrons qu'il existe une solution unique et qu'elle s'exprime à l'aide d'un noyau stochastique relié au problème. Cette formulation nous permet d'étudier les propriétés de base de la solution, telles que la continuité et le comportement au bord, en utilisant le calcul de Malliavin. () 2002 Éditions scientifiques et médicales Elsevier SAS

\section{Introduction}

Our purpose in this paper is to study stochastic partial differential equations of the form

$$
\left\{\begin{array}{l}
d_{t} u(t, x)=\frac{\partial^{2} u}{\partial x^{2}}(t, x) \mathrm{d} t+\sum_{j=1}^{n}\left[b_{j}(x) \frac{\partial u}{\partial x}(t, x)+F_{j}(t, x, u(t, x))\right] \mathrm{d} W_{t}^{j} \\
u(t, 0)=\dot{V}_{t} \\
u(0, x)=0, \quad(t, x) \in I^{T}:=[0, T] \times \mathbb{R}_{+}
\end{array}\right.
$$


where $W_{t}=\left(W_{t}^{1}, \ldots, W_{t}^{n}\right)$ is a real standard $n$-dimensional Wiener process and $V_{t}$ is a Brownian motion adapted to the filtration generated by $W_{t}$. We set the initial condition equal to zero in order to simplify the notation; no major problems arise in the general case.

Existence and properties of the solution to an evolution problem with boundary noise are broadly investigated in the literature. The way we interpret the solution $u(t, x)$, see Definition 2, is in particular inspired from that given in [16]. An alternative approach we would like to mention is given by the semigroup techniques developed, among others, in [4] and [11], which allow to study the global properties of the solution.

In this paper we show that the solution of Eq. (1.1) is the random process $u(t, x)$ which satisfies the evolution equation

$$
\begin{aligned}
u(t, x)= & \int_{0}^{t} \frac{\partial p_{D}}{\partial y}(s, t, 0, x) \mathrm{d} V_{s} \\
& +\sum_{j=1}^{n} \int_{0}^{t}\left(\int_{\mathbb{R}_{+}} p_{D}(s, t, y, x) F_{j}(s, y, u(s, y)) \mathrm{d} y\right) \mathrm{d} W_{s}^{j},
\end{aligned}
$$

where $p_{D}(s, t, y, x)$ is the fundamental solution of the linear homogeneous part of Eq. (1.1). This kernel is adapted to $\mathcal{F}_{s}^{t}=\sigma\left\{W_{r}, s \leqslant r \leqslant t\right\}$, which means that the integrals in Eq. (1.2) are anticipative. We will interpret the first integral in the backward Itô sense and the second one in the Skorohod sense (see [15]).

The evolution equation related to problem (1.1) in the whole space has been studied in [14] and in [2]. Following the approach introduced in these papers we establish the existence and uniqueness of a solution to Eq. (1.2) with values in an appropriate weighted Sobolev space, which turns to be the space $L_{\gamma}^{p}$ of real-valued functions $f$ such that

$$
\int_{\mathbb{R}_{+}}|f(x)|^{p}\left(x^{p-1+\gamma} \wedge 1\right) \mathrm{d} x<\infty,
$$

where $0<\gamma<1$ and $p \geqslant 2$. The main theorem is a consequence of estimates on Skorohod integrals of the form

$$
\int_{0}^{t}\left(\int_{\mathbb{R}_{+}} p_{D}(s, t, y, x) \Phi(s, y) \mathrm{d} y\right) \mathrm{d} W_{s},
$$

obtained using the techniques of Malliavin calculus; by means of such estimates we show also that the solution of Eq. (1.2) is continuous in the open half-line $\{x>0\}$. We are interested in the regularity behavior of the solution, especially near the origin, where the boundary data is highly irregular. Our results are, again, connected to those proved in [16]; we will show that near the boundary the solution still behaves not too badly: namely, it has a singularity of order 1 in $x=0$ and for any $\alpha>0$ it holds that $x^{1+\alpha} u(t, x) \rightarrow 0$ a.s. as $x \downarrow 0$. 


\subsection{Main assumptions and statement of the results}

In this paper, we will always assume without stating that the coefficients in Eq. (1.1) satisfy the following regularity assumptions:

(h1). The drift coefficients $b_{j}: \mathbb{R}_{+} \rightarrow \mathbb{R}$ belong to $C_{b}^{3}$ and they satisfy the joint ellipticity condition

$$
\Sigma(x):=1-\frac{1}{2} \sum_{j=1}^{n} b_{j}^{2}(x) \geqslant \varepsilon>0 .
$$

(h2). The nonlinear terms $F_{j}(t, x, u)$ are uniformly Lipschitz continuous in the variable $u$ : there exists a constant $L$ such that for all $t \in[0, T], x \in \mathbb{R}$ and $j=1, \ldots, n$

$$
\left|F_{j}(t, x, u)-F_{j}(t, x, v)\right| \leqslant L|u-v| .
$$

$(F)_{p, \vartheta}$. We say that condition $(F)_{p, \vartheta}$ holds, where $p \geqslant 2,0<\vartheta<1$, if there exists a function $h(x) \in L_{\vartheta}^{p}$ such that for all $t \in[0, T]$ and $j=1, \ldots, n$

$$
\left|F_{j}(t, x, 0)\right| \leqslant h(x) \quad \text { for every } x \in \mathbb{R}_{+} .
$$

The class of functions which satisfy condition $(F)_{p, \vartheta}$ is quite spread; for example, we can take $h(x)=1 / x$, which belongs to $L_{\vartheta}^{p}$ for any $p \geqslant 2,0<\vartheta<1$.

We make use of the following notation. Let $(\Omega, \mathcal{F}, \mathbb{P})$ be the canonical probability space of the $n$-dimensional Brownian motion $W=\left\{W_{t}, t \in[0, T]\right\}$. Given a Banach space $X$, we denote $L^{p}(\Omega \times[0, T] ; X)$ the space of $p$-integrable random processes with values in $X$ and $M^{p}(\Omega \times[0, T] ; X)$ the subspace of $p$-integrable, adapted processes. The following result states the main properties of the solution to the evolution equation (1.2). The proof will be given in Section 4.

THEOREM 1. - Assume that condition $(F)_{p, \vartheta}$ holds for some $p \geqslant 2,0<\vartheta<1$. Then Eq. (1.2) has a unique solution $u(t, x)$ in $M^{p}\left(\Omega \times[0, T] ; L_{\gamma}^{p}\right)$ for any $\gamma \in(0,1)$. If moreover condition $(F)_{p, \vartheta}$ holds for some $p>2,0<\vartheta<1$, then the function $u(t, \cdot)$ is continuous on $[\delta, \infty)$ for every $\delta>0$ and it satisfies that

$$
x^{1+\alpha} u(t, x) \rightarrow 0 \quad \text { a.s. }
$$

for every $\alpha>0$.

Our next aim is to properly define the meaning of weak solution for Eq. (1.1). We consider the class of smooth functions $f(t, x)$ which belong to $C_{0}^{\infty}$ and satisfy $f(t, 0)=0$; a formal computation leads to

$$
\begin{gathered}
\int_{0}^{\infty} \int_{\mathbb{R}_{+}} u(s, x)\left[\frac{\partial f}{\partial s}(s, x)+\frac{\partial^{2} f}{\partial x^{2}}(s, x)\right] \mathrm{d} x \mathrm{~d} s+\sum_{j=1}^{n} \int_{0}^{\infty} \int_{\mathbb{R}_{+}} F_{j}(s, x, u(s, x)) f(s, x) \mathrm{d} x \mathrm{~d} W_{s}^{j} \\
-\sum_{j=1}^{n} \int_{0}^{\infty} \int_{\mathbb{R}_{+}} \frac{\partial}{\partial x}\left[b_{j}(x) f(s, x)\right] u(s, x) \mathrm{d} x \mathrm{~d} W_{s}^{j}=-\int_{0}^{\infty} \frac{\partial f}{\partial x}(s, 0) \mathrm{d} V_{s} .
\end{gathered}
$$


To make this rigorous, we need to give a meaning to the left hand side of the previous equation, because $u(t, x)$ might not exist on the boundary. However, we can "avoid" the boundary by considering $x+\varepsilon$ instead of $x$ as the space variable in (1.6).

Definition 2. - A function $u=u(t, x)$ in $L^{p}\left(\Omega \times[0, T] ; L_{\gamma}^{p}\right)$, continuous in $x \in$ $(0,+\infty)$, is a weak solution of Eq. (1.1) if the following identity holds

$$
\begin{aligned}
\lim _{\varepsilon \rightarrow 0} & \left\{\int_{0}^{\infty} \int_{\mathbb{R}_{+}} u(s, x+\varepsilon)\left[\frac{\partial f}{\partial s}(s, x)+\frac{\partial^{2} f}{\partial x^{2}}(s, x)\right] \mathrm{d} x \mathrm{~d} s\right. \\
& +\sum_{j=1}^{n} \int_{0}^{\infty} \int_{\mathbb{R}_{+}} F_{j}(s, x+\varepsilon, u(s, x+\varepsilon)) f(s, x) \mathrm{d} x \mathrm{~d} W_{s}^{j} \\
& \left.-\sum_{j=1}^{n} \int_{0}^{\infty} \int_{\mathbb{R}_{+}} \frac{\partial}{\partial x}\left[b_{j}(x+\varepsilon) f(s, x)\right] u(s, x+\varepsilon) \mathrm{d} x \mathrm{~d} W_{s}^{j}\right\}=-\int_{0}^{\infty} \frac{\partial f}{\partial x}(s, 0) \mathrm{d} V_{s} .
\end{aligned}
$$

In Section 5 we will prove the following result.

THEOREM 3. - Assume that condition $(F)_{p, \vartheta}$ holds for some $p>2,0<\vartheta<1$. Then the solution $u(t, x) \in M^{p}\left(\Omega \times[0, T] ; L_{\gamma}^{p}\right)$ of Eq. (1.2) given in the previous theorem is the unique weak solution of Eq. (1.1).

\section{The construction of the stochastic kernel}

In this section we recall the definition and the basic properties of the stochastic kernel related with Eq. (1.1). This construction follows that in [14]; see also [8]. In order to make the paper self-contained, we recall first the basic definitions of Malliavin calculus required along the paper. For all the details we refer to the monograph [12].

\subsection{Basic definitions in Malliavin calculus}

Let $H$ be the Hilbert space $L^{2}\left([0, T] ; \mathbb{R}^{n}\right)$. For any $h \in H$ we denote by $W(h)$ the Wiener integral $W(h)=\sum_{j=1}^{n} \int_{0}^{T} h_{j}(t) \mathrm{d} W_{t}^{j}$. Let $\mathcal{S}$ be the set of smooth and cylindrical random variables of the form

$$
F=f\left(W\left(h^{1}\right), \ldots, W\left(h^{m}\right)\right),
$$

where $n \geqslant 1, f \in C_{b}^{\infty}\left(\mathbb{R}^{m}\right)$ ( $f$ and all its derivatives are bounded), and $h^{1}, \ldots, h^{m} \in H$. Given a random variable $F$ of the form (2.1), we define its derivative as the stochastic $n$-dimensional process $\left\{D_{t} F, t \in[0, T]\right\}$ given by

$$
D_{t} F=\sum_{i=1}^{m} \frac{\partial f}{\partial x_{i}}\left(W\left(h^{1}\right), \ldots, W\left(h^{m}\right)\right) h^{i}(t), \quad t \in[0, T] .
$$

In the same way, we can define the iterated derivative operator on a cylindrical random variable by setting

$$
D_{t_{1}, \ldots, t_{p}}^{p} F=D_{t_{1}} \ldots D_{t_{p}} F
$$


The derivative operator $D^{p}$ is a closable unbounded operator from $L^{2}(\Omega ; H)$ into $L^{2}\left(\Omega ; H^{p}\right)$ for each $p \geqslant 1$. We denote by $\mathbb{D}^{p, 2}$ the closure of $\mathcal{S}$ with respect to the norm defined by

$$
\|F\|_{p, 2}^{2}=\|F\|_{L^{2}(\Omega)}^{2}+\sum_{k=1}^{p}\left\|D^{k} F\right\|_{L^{2}\left(\Omega ; H^{k}\right)}^{2} .
$$

Next, we introduce the adjoint of the derivative operator $D$; it is the Skorohod integral with respect to the Brownian motion $W$ :

$$
\delta(u)=\sum_{j=1}^{n} \int_{0}^{T} u_{j}(t) \mathrm{d} W_{t}^{j}
$$

for any $u \in \operatorname{Dom}(\delta) \subset L^{2}\left(\Omega \times[0, T] ; \mathbb{R}^{n}\right)$. The domain of the Skorohod integral contains the set $M^{2}(\Omega ; H)$ of square integrable and adapted processes and the operator $\delta$ restricted to this space coincides with the Itô stochastic integral (see [13]).

Let us define the family of spaces $\mathbb{L}^{n, 2}=L^{2}\left(H ; \mathbb{D}^{n, 2}\right)$ equipped with the norm

$$
\|v\|_{n, 2}^{2}=\|v\|_{L^{2}(\Omega ; H)}^{2}+\sum_{k=1}^{p}\left\|D^{k} v\right\|_{L^{2}\left(\Omega ; H^{k+1}\right)}^{2} .
$$

We recall that $\mathbb{L}^{1,2}$ is included in the domain of $\delta$, and for a process $u$ in $\mathbb{L}^{1,2}$ we can compute the variance of the Skorohod integral of $u$ as follows

$$
\mathbb{E}\left(\delta(u)^{2}\right)=\mathbb{E} \int_{0}^{T}\left|u_{t}\right|^{2} \mathrm{~d} t+\mathbb{E} \int_{0}^{T} \int_{0}^{T}\left\langle D_{s} u_{t}, D_{t} u_{s}\right\rangle \mathrm{d} s \mathrm{~d} t .
$$

Main tools from Malliavin calculus that we use in the paper are contained in [12]. Apart from these, we need to recall the following change-of-variables formula for the Skorohod integral; see [2] and [7].

Proposition 4. - Consider a process of the form $X_{t}=\sum_{j=1}^{n} \int_{0}^{t} u_{j}(s) \mathrm{d} W_{s}^{j}$, where (a1) $u \in \mathbb{L}^{2,2}$,

(a2) $u \in L^{\beta}\left(\Omega \times[0, T] ; \mathbb{R}^{n}\right)$, for some $\beta>2$,

(a3) $\int_{0}^{T}|u(s)|^{2} \mathrm{~d} s<N$ for some positive constant $N$.

Let $F: \mathbb{R} \rightarrow \mathbb{R}$ be a twice continuously differentiable function such that $F^{\prime \prime}$ is bounded. Then we have

$$
\begin{aligned}
F\left(X_{t}\right)= & F(0)+\sum_{j=1}^{n} \int_{0}^{t} F^{\prime}\left(X_{s}\right) u_{j}(s) \mathrm{d} W_{s}^{j}+\frac{1}{2} \int_{0}^{t} F^{\prime \prime}\left(X_{s}\right)|u(s)|^{2} \mathrm{~d} s \\
& +\int_{0}^{t} F^{\prime \prime}\left(X_{s}\right)\left\langle u(s), \sum_{j=1}^{n} \int_{0}^{s} D_{s}^{j} u(r) \mathrm{d} W_{r}^{j}\right\rangle \mathrm{d} s .
\end{aligned}
$$




\subsection{The stochastic heat kernel}

Let $B=\left\{B_{t}, t \in[0, T]\right\}$ be a Brownian motion with variance $2 t$ defined on another probability space $(\mathcal{W}, \mathcal{G}, \mathbb{Q})$. Consider the following backward stochastic differential equation on the probability space $(\Omega \times \mathcal{W}, \mathcal{F} \otimes \mathcal{G}, \mathbb{P} \times \mathbb{Q})$ :

$$
\varphi_{t, s}(x)=x-\sum_{j=1}^{n} \int_{s}^{t} b_{j}\left(\varphi_{t, r}(x)\right) \mathrm{d} W_{r}^{j}+\int_{s}^{t} \sqrt{\Sigma\left(\varphi_{t, r}(x)\right)} \mathrm{d} B_{r} .
$$

From the results in Theorems 3.4.1 and 4.5.1 of [9] we know that Eq. (2.3) has a solution $\varphi=\left\{\varphi_{t, s}(x), 0 \leqslant s \leqslant t \leqslant T, x \in \mathbb{R}\right\}$, continuous in the three variables and verifying

$$
\varphi_{r, s}\left(\varphi_{t, r}(x)\right)=\varphi_{t, s}(x)
$$

for all $s<r<t, x \in \mathbb{R}$.

The existence of the kernel for the operator $\frac{\partial^{2}}{\partial x^{2}}+\sum_{j=1}^{n} b_{j}(x) \dot{W}_{t}^{j} \frac{\partial}{\partial x}$ is proved in Proposition 9 of [14].

PROPOSITION 5. - Let $\varphi_{t, s}(x)$ be the stochastic flow defined by Eq. (2.3). Then there is a version of the marginal density $p(s, t, y, x)=\frac{Q\left[\varphi_{t, s}(x) \in \mathrm{d} y\right]}{\mathrm{d} y}$ which is $\mathcal{F}_{s}^{t}$-adapted and it satisfies the semigroup property:

$$
p(s, t, x, y)=\int_{\mathbb{R}} p(s, r, y, z) p(r, t, z, x) \mathrm{d} z,
$$

for all $0 \leqslant s<r<t \leqslant T, x, y \in \mathbb{R}$.

We denote $q(s, t, y, x)$ the heat kernel on $\mathbb{R}$ defined by the Laplace operator $\frac{\partial^{2}}{\partial x^{2}}$, which is known to be

$$
q(s, t, y, x)=\frac{1}{2 \sqrt{\pi(t-s)}} \exp \left(-\frac{(y-x)^{2}}{4(t-s)}\right) .
$$

We set $q_{D}(s, t, y, x)=q(s, t, y, x)-q(s, t,-y, x)$ : it is known that $q_{D}(s, t, y, x)$ is the heat kernel on $\mathbb{R}_{+}$with zero Dirichlet boundary conditions. We recall that $\frac{\partial q}{\partial y}(s, \cdot, y, x)$ is the density function of a Brownian passage time, which also means that for any $x, z \in \mathbb{R}_{+}$

$$
\int_{s}^{\infty}\left[q_{D}(s, t, y, x)-q_{D}(s, t, y, z)\right] \mathrm{d} t=0 .
$$

This can be easily proved as follows:

$$
\begin{aligned}
& \int_{s}^{\infty} {\left[q_{D}(s, t, y, x)-q_{D}(s, t, y, z)\right] \mathrm{d} t } \\
&=\int_{s}^{\infty} \int_{-y}^{y}\left[\frac{\partial q}{\partial y}(s, t, \eta, x)-\frac{\partial q}{\partial y}(s, t, \eta, z)\right] \mathrm{d} \eta \mathrm{d} t
\end{aligned}
$$




$$
=\int_{-y}^{y}\left[\int_{s}^{\infty} \frac{\partial q}{\partial y}(s, t, \eta, x) \mathrm{d} t-\int_{s}^{\infty} \frac{\partial q}{\partial y}(s, t, \eta, z) \mathrm{d} t\right] \mathrm{d} \eta=0 .
$$

Consider now the stochastic kernel $p_{D}(s, t, y, x)=p(s, t, y, x)-p(s, t,-y, x)$. From the analog result for the kernel $p$, that is given in Eq. (4.15) of [14], it is possible to prove the backward Kolmogorov equation for any test function $f \in C_{0}^{\infty}(\mathbb{R})$ :

$$
\begin{aligned}
\int_{\mathbb{R}_{+}} p_{D}(s, t, y, x) f(y) \mathrm{d} y= & f(x)+\sum_{j=1}^{n} \int_{s}^{t} b_{j}(x)\left(\int_{\mathbb{R}_{+}} \frac{\partial p_{D}}{\partial x}(s, r, y, x) f(y) \mathrm{d} y\right) \mathrm{d} W_{r}^{j} \\
& +\int_{s}^{t}\left(\int_{\mathbb{R}_{+}} \frac{\partial^{2} p_{D}}{\partial x^{2}}(s, r, y, x) f(y) \mathrm{d} y\right) \mathrm{d} r
\end{aligned}
$$

This gives us the evolution equation

$$
\begin{aligned}
p_{D}(s, t, y, x)= & q_{D}(s, t, y, x) \\
& +\sum_{j=1}^{n} \int_{s}^{t}\left(\int_{\mathbb{R}_{+}} b_{j}(z) q_{D}(r, t, z, x) \frac{\partial p_{D}}{\partial z}(s, r, y, z) \mathrm{d} z\right) \mathrm{d} W_{r}^{j},
\end{aligned}
$$

from which we can prove that this kernel satisfies the semigroup property

$$
p_{D}(s, t, y, x)=\int_{\mathbb{R}_{+}} p_{D}(s, r, y, u) p_{D}(r, t, u, x) \mathrm{d} u
$$

and the Dirichlet boundary conditions.

In order to study the stochastic problem (1.1) it is important to have estimates on the kernel $p_{D}(s, t, y, x)$. In the sequel $C$ and $c$ will be some positive constants that can change from line to line.

LEMmA 6. - For all $s<t, x, y \in \mathbb{R}_{+}$, it holds that

$$
\begin{gathered}
\left\|p_{D}(s, t, y, x)\right\|_{L^{p}(\Omega)} \leqslant C(t-s)^{-1 / 2} \exp \left(-\frac{|y-x|^{2}}{c(t-s)}\right), \\
\left\|\frac{\partial^{m+k} p_{D}}{\partial y^{k} \partial x^{m}}(s, t, y, x)\right\|_{L^{p}(\Omega)} \leqslant C(t-s)^{-(m+k+1) / 2} \exp \left(-\frac{|y-x|^{2}}{c(t-s)}\right)
\end{gathered}
$$

for each $m=0,1,2, k=0,1$ and for some constants $C, c>0$.

Proof. - In Proposition 11 of [14] some estimates for the kernel $p(s, t, y, x)$ are given. The first part of the lemma is proved using those results; here we give only the proof of (2.11) in the case $m=k=1$, in which some new ideas are needed.

We observe that the chain rule for the Malliavin derivative gives

$$
D\left(\mathbf{1}_{[y, \infty)}(F)\right)=\frac{\partial}{\partial x}\left(\mathbf{1}_{[y, \infty)}(F)\right) D F=-\frac{\partial}{\partial y}\left(\mathbf{1}_{[y, \infty)}(F)\right) D F .
$$


Using the integration-by-parts formula of Malliavin calculus we can write

$$
\frac{\partial p}{\partial x}(s, t, y, x)=\mathbb{E}_{Q}\left[\mathbf{1}_{\left\{\varphi_{t, s}(x)>y\right\}} \delta\left(\delta\left(\frac{\varphi_{t, s}^{\prime}(x) D \varphi_{t, s}(x)}{\left\|D \varphi_{t, s}(x)\right\|^{2}}\right) \frac{D \varphi_{t, s}(x)}{\left\|D \varphi_{t, s}(x)\right\|^{2}}\right)\right],
$$

from where it follows that

$$
\begin{aligned}
& \frac{\partial^{2} p}{\partial x \partial y}(s, t, y, x) \\
& \quad=-\mathbb{E}_{Q}\left[\mathbf{1}_{\left\{\varphi_{t, s}(x)>y\right\}} \delta\left(\delta\left(\delta\left(\frac{\varphi_{t, s}^{\prime}(x) D \varphi_{t, s}(x)}{\left\|D \varphi_{t, s}(x)\right\|^{2}}\right) \frac{D \varphi_{t, s}(x)}{\left\|D \varphi_{t, s}(x)\right\|^{2}}\right) \frac{D \varphi_{t, s}(x)}{\left\|D \varphi_{t, s}(x)\right\|^{2}}\right)\right] .
\end{aligned}
$$

Denote

$$
\begin{gathered}
H_{0}=\delta\left(\frac{\varphi_{t, s}^{\prime}(x) D \varphi_{t, s}(x)}{\left\|D \varphi_{t, s}(x)\right\|^{2}}\right), \\
H_{i}=\delta\left(\frac{D \varphi_{t, s}(x)}{\left\|D \varphi_{t, s}(x)\right\|^{2}} H_{i-1}\right), \quad i=1,2, \ldots
\end{gathered}
$$

With this notation we can write

$$
\frac{\partial^{2} p}{\partial x \partial y}(s, t, y, x)=-\mathbb{E}_{Q}\left[\mathbf{1}_{\left\{\varphi_{t, s}(x)>y\right\}} H_{2}\right] .
$$

We define moreover the process $B_{t, s}(x)=\varphi_{t, s}(x)-x$. As a first step we study estimates on the indicator function. It follows that

$$
\frac{\partial^{2} p}{\partial x \partial y}(s, t, y, x)=-\mathbb{E}_{Q}\left[\mathbf{1}_{\left\{B_{t, s}(x)>y-x\right\}} H_{2}\right]=-\mathbb{E}_{Q}\left[\mathbf{1}_{\left\{-B_{t, s}(x)>x-y\right\}} H_{2}\right] ;
$$

since $B$ and $-B$ have the same distribution, it is sufficient to study one case. Assume $y>x$ : using the trivial bound

$$
\mathbf{1}_{\left\{B_{t, s}>a\right\}} \leqslant \exp \left(\frac{K B_{t, s}(x)^{2}}{p(t-s)}\right) \exp \left(-\frac{K a^{2}}{p(t-s)}\right)
$$

valid for any $a \geqslant 0, K>0$, we obtain

$$
\left|\frac{\partial^{2} p}{\partial x \partial y}(s, t, y, x)\right| \leqslant \exp \left(-\frac{K|x-y|^{2}}{p(t-s)}\right) \mathbb{E}_{Q}\left[\exp \left(\frac{K B_{t, s}(x)^{2}}{p(t-s)}\right)\left|H_{2}\right|\right] .
$$

By Schwarz's inequality

$$
\mathbb{E}\left[\exp \left(\frac{K B_{t, s}(x)^{2}}{p(t-s)}\right)\left|H_{2}\right|\right]^{p} \leqslant\left(\mathbb{E}\left[\exp \left(\frac{2 K B_{t, s}(x)^{2}}{(t-s)}\right)\right] \mathbb{E}\left|H_{2}\right|^{2 p}\right)^{1 / 2} .
$$

The following estimate is proved in [2]

$$
\mathbb{E}\left[\exp \left(\frac{2 K B_{t, s}(x)^{2}}{(t-s)}\right)\right]=\frac{1}{\sqrt{1-8 K}}
$$


with the same arguments as in the proof of Proposition 11 of [14], we can prove that

$$
\left(\mathbb{E}\left|H_{2}\right|^{2 p}\right)^{1 / 2 p} \leqslant C(t-s)^{-3 / 2}
$$

which yields the desired estimate.

Remark 7. - From the definition of $p_{D}(s, t, y, x)=p(s, t, y, x)-p(s, t,-y, x)$ and using the mean value theorem we may prove the estimate

$$
\left\|p_{D}(s, t, y, x)\right\|_{L^{p}(\Omega)} \leqslant C y^{a}(t-s)^{-\frac{1}{2}-\frac{a}{2}} \exp \left(-\frac{(y-x)^{2}}{c(t-s)}\right)
$$

for $0 \leqslant a \leqslant 1$.

The following lemma is concerned with the Malliavin derivative of the stochastic kernel. The operator $D^{-}$defined in (2.12) is usually known in the literature as the forward Malliavin derivative.

Lemma 8 . - For all $0 \leqslant s<t \leqslant T, x, y \in \mathbb{R}_{+}, p_{D}(s, t, x, y) \in \mathbb{D}^{1,2}$ and there exists a version of the derivative such that the following limit exists in $L^{2}\left(\Omega \times \mathbb{R}_{+} ; \mathbb{R}^{n}\right)$ for every $s, t \in[0, T]$ and $x \in \mathbb{R}_{+}$:

$$
D_{s}^{-} p_{D}(s, t, \cdot, x)=\lim _{\varepsilon \downarrow 0} D_{s} p_{D}(s-\varepsilon, t, \cdot, x) .
$$

Moreover, it satisfies the evolution equation

$$
D_{s} p_{D}(r, t, y, x)=\int_{\mathbb{R}_{+}}\left(D_{s}^{-} p_{D}(s, t, u, x)\right) p_{D}(r, s, y, u) \mathrm{d} u
$$

and the estimates

$$
\begin{gathered}
\left\|D_{s}^{-} p_{D}(s, t, y, x)\right\|_{L^{p}(\Omega)} \leqslant C(t-s)^{-1} \exp \left(-\frac{|y-x|^{2}}{c(t-s)}\right), \\
\left\|D_{s}^{-}\left[p_{D}(s, t, y, x)-p_{D}(s, t, y, z)\right]\right\|_{L^{p}(\Omega)} \\
\leqslant(t-s)^{-1-\sigma / 2}|z-x|^{\sigma}\left[\exp \left(-\frac{|y-x|^{2}}{c(t-s)}\right)+\exp \left(-\frac{\mid y-z^{2}}{c(t-s)}\right)\right],
\end{gathered}
$$

for $0 \leqslant \sigma \leqslant 1$.

Proof. - The regularity properties of $p_{D}(s, t, x, y)$ follows from those of $p(s, t, x, y)$ that are proved in [14]. Using the semigroup property (2.9) we get

$$
D_{s} p_{D}(r, t, y, x)=D_{s} \int_{\mathbb{R}_{+}} p_{D}(s-\varepsilon, t, u, x) p_{D}(r, s-\varepsilon, y, u) \mathrm{d} u .
$$

Now Eq. (2.13) follows recalling that $p_{D}(s, t, x, y)$ is $\mathcal{F}_{s}^{t}$-adapted and passing to the limit $\varepsilon \rightarrow 0$. From the backward Kolmogorov equation (2.7) we can prove the following 
representation for the derivative of $\int_{\mathbb{R}_{+}} p_{D}(s, t, y, x) f(y) \mathrm{d} y$, see for example Section 3 of [3]

$$
D_{s} \int_{\mathbb{R}_{+}} p_{D}(r, t, y, x) f(y) \mathrm{d} y=\int_{\mathbb{R}_{+}} p_{D}(r, s, y, x) b(y) \frac{\partial}{\partial y}\left(\int_{\mathbb{R}_{+}} p_{D}(s, t, u, y) f(u) \mathrm{d} u\right) \mathrm{d} y .
$$

Then it follows that

$$
\begin{aligned}
& D_{s} \int_{\mathbb{R}_{+}} p_{D}(s-\varepsilon, t, y, x) f(y) \mathrm{d} y \\
& \quad=\int_{\mathbb{R}_{+}} p_{D}(s-\varepsilon, s, y, x) b(y) \frac{\partial}{\partial y}\left(\int_{\mathbb{R}_{+}} p_{D}(s, t, z, y) f(z) \mathrm{d} z\right) \mathrm{d} y
\end{aligned}
$$

and letting $\varepsilon$ tend to zero we obtain

$$
D_{s}^{-} p_{D}(s, t, y, x)=-b(x) \frac{\partial}{\partial x} p_{D}(s, t, y, x) .
$$

Now, using the estimates of the previous lemma the result follows.

\section{The boundary term}

We consider here the term

$$
\Psi(t, x):=\int_{0}^{t} \frac{\partial p_{D}}{\partial y}(s, t, 0, x) \mathrm{d} V_{s}
$$

It is easy to prove that $\Psi \in M^{p}\left(\Omega \times[0, T] ; L_{\gamma}^{p}\right)$ for every $p \geqslant 2,0<\gamma<1$. In fact we have, using estimate (2.11) and the isometry property of the Itô backward integral, that

$$
\begin{aligned}
\mathbb{E}\left|\int_{0}^{t} \frac{\partial p_{D}}{\partial y}(s, t, 0, x) \mathrm{d} V_{s}\right|^{p} & \leqslant C\left|\int_{0}^{t}(t-s)^{-2} \exp \left(-\frac{x^{2}}{c(t-s)}\right) \mathrm{d} s\right|^{p / 2} \\
& =C\left|\frac{1}{x^{2}} \mathrm{e}^{-x^{2} / t}\right|^{p / 2}=C x^{-p} \mathrm{e}^{-p x^{2} / 2 t}
\end{aligned}
$$

The following lemma will be necessary in Section 5 .

LEMmA 9. - For every test function $\varphi \in C_{0}^{\infty}([0, T])$ the following convergence holds

$$
\int_{0}^{T} \Psi(s, \varepsilon) \varphi(s) \mathrm{d} s \underset{\varepsilon \rightarrow 0}{\longrightarrow} \int_{0}^{T} \varphi(r) \mathrm{d} V_{r} \quad \text { a.s. }
$$


Proof. - In order to prove the almost sure convergence we notice that, since $\varepsilon>0$, we can apply Fubini's theorem and study the limit

$$
\int_{0}^{T}\left(\int_{r}^{T} \frac{\partial p_{D}}{\partial y}(r, s, 0, \varepsilon) \varphi(s) \mathrm{d} s\right) \mathrm{d} V_{r} \rightarrow \int_{0}^{T} \varphi(r) \mathrm{d} V_{r} .
$$

Notice that it is sufficient to prove that

$$
f(\varepsilon)=\int_{0}^{T} \int_{r}^{T} \frac{\partial p_{D}}{\partial y}(r, s, 0, \varepsilon) \varphi(s) \mathrm{d} s \mathrm{~d} V_{r}
$$

is a continuous function in 0 , and to identify the limit.

In order to study the continuity of $f$ notice that for every $\varepsilon, \varepsilon^{\prime}>0$

$$
f(\varepsilon)-f\left(\varepsilon^{\prime}\right)=\int_{0}^{T} \int_{r}^{T}\left[\frac{\partial p_{D}}{\partial y}(r, s, 0, \varepsilon)-\frac{\partial p_{D}}{\partial y}\left(r, s, 0, \varepsilon^{\prime}\right)\right] \varphi(s) \mathrm{d} s \mathrm{~d} V_{r}
$$

so we reduce to estimate the integral

$$
I_{r}\left(\varepsilon, \varepsilon^{\prime}\right)=\int_{r}^{T}\left[\frac{\partial p_{D}}{\partial y}(r, s, 0, \varepsilon)-\frac{\partial p_{D}}{\partial y}\left(r, s, 0, \varepsilon^{\prime}\right)\right] \varphi(s) \mathrm{d} s
$$

As $\varphi$ has compact support we can write the above integral as

$$
I_{r}\left(\varepsilon, \varepsilon^{\prime}\right)=\int_{r}^{\infty}\left[\frac{\partial p_{D}}{\partial y}(r, s, 0, \varepsilon)-\frac{\partial p_{D}}{\partial y}\left(r, s, 0, \varepsilon^{\prime}\right)\right] \varphi(s) \mathrm{d} s
$$

and the following identity is obvious

$$
\begin{aligned}
I_{r}\left(\varepsilon, \varepsilon^{\prime}\right)= & \int_{r}^{\infty}\left[\frac{\partial p_{D}}{\partial y}(r, s, 0, \varepsilon)-\frac{\partial p_{D}}{\partial y}\left(r, s, 0, \varepsilon^{\prime}\right)\right][\varphi(s)-\varphi(r)] \mathrm{d} s \\
& +\varphi(r) \int_{r}^{\infty}\left[\frac{\partial p_{D}}{\partial y}(r, s, 0, \varepsilon)-\frac{\partial p_{D}}{\partial y}\left(r, s, 0, \varepsilon^{\prime}\right)\right] \mathrm{d} s \\
= & I_{1}\left(\varepsilon, \varepsilon^{\prime}\right)+I_{2}\left(\varepsilon, \varepsilon^{\prime}\right) .
\end{aligned}
$$

We start from the first term. As $\varphi$ is smooth we can estimate the first term as follows

$$
\begin{aligned}
\mathbb{E}\left|I_{1}\left(\varepsilon, \varepsilon^{\prime}\right)\right|^{p} & \leqslant C\left|\int_{r}^{T}\left\|\frac{\partial p_{D}}{\partial y}(r, s, 0, \varepsilon)-\frac{\partial p_{D}}{\partial y}\left(r, s, 0, \varepsilon^{\prime}\right)\right\|_{L^{p}(\Omega)}(s-r) \mathrm{d} s\right|^{p} \\
& \leqslant C\left|\varepsilon-\varepsilon^{\prime}\right|^{p} \mid \int_{r}^{T}(s-r)^{-1 / 2}
\end{aligned}
$$




$$
\begin{aligned}
& \times\left.\left[\exp \left(-\frac{\varepsilon^{2}}{c(s-r)}\right)+\exp \left(-\frac{\varepsilon^{\prime 2}}{c(s-r)}\right)\right] \mathrm{d} s\right|^{p} \\
\leqslant & C\left|\varepsilon-\varepsilon^{\prime}\right|^{p} .
\end{aligned}
$$

On the other hand, using the evolution equation (2.8) we can write

$$
\begin{aligned}
\int_{r}^{\infty} & {\left[\frac{\partial p_{D}}{\partial y}(r, s, 0, \varepsilon)-\frac{\partial p_{D}}{\partial y}\left(r, s, 0, \varepsilon^{\prime}\right)\right] \mathrm{d} s } \\
= & \frac{\partial}{\partial y} \int_{r}^{\infty}\left[p_{D}(r, s, 0, \varepsilon)-p_{D}\left(r, s, 0, \varepsilon^{\prime}\right)\right] \mathrm{d} s \\
= & \frac{\partial}{\partial y} \int_{r}^{\infty}\left[q_{D}(r, s, 0, \varepsilon)-q_{D}\left(r, s, 0, \varepsilon^{\prime}\right)\right] \mathrm{d} s \\
& +\sum_{j=1}^{n} \frac{\partial}{\partial y} \int_{r}^{\infty}\left[\int _ { r } ^ { s } \left(\int_{\mathbb{R}_{+}}\left[q_{D}(u, s, z, \varepsilon)-q_{D}\left(u, s, z, \varepsilon^{\prime}\right)\right]\right.\right. \\
& \left.\left.\times \frac{\partial p_{D}}{\partial z}(r, u, 0, z) b_{j}(z) \mathrm{d} z\right) \mathrm{~d} W_{u}^{j}\right] \mathrm{d} s .
\end{aligned}
$$

Now we need to apply Fubini's theorem to the right-hand side of the previous expression, which becomes equal to

$$
\begin{aligned}
& \frac{\partial}{\partial y} \int_{r}^{\infty}\left[q_{D}(r, s, 0, \varepsilon)-q_{D}\left(r, s, 0, \varepsilon^{\prime}\right)\right] \mathrm{d} s \\
& \quad+\sum_{j=1}^{n} \frac{\partial}{\partial y} \int_{r}^{\infty}\left[\int_{\mathbb{R}_{+}}\left[\int_{u}^{\infty}\left[q_{D}(u, s, z, \varepsilon)-q_{D}\left(u, s, z, \varepsilon^{\prime}\right)\right] \mathrm{d} s\right]\right. \\
& \left.\quad \times \frac{\partial p_{D}}{\partial z}(r, u, 0, z) b_{j}(z) \mathrm{d} z\right] \mathrm{d} W_{u}^{j} .
\end{aligned}
$$

The identity in Eq. (2.6) applies in both integrals, so that $I_{2}\left(\varepsilon, \varepsilon^{\prime}\right)=0$, which means that

$$
\mathbb{E}\left|I_{r}\left(\varepsilon, \varepsilon^{\prime}\right)\right|^{p} \leqslant C\left|\varepsilon-\varepsilon^{\prime}\right|^{p} .
$$

By Kolmogorov's continuity theorem this implies that there exists a version of $f(\varepsilon)$ that is continuous, with sample paths that are a.s. Hölder continuous functions with an arbitrary exponent less that $\frac{p-1}{p}$. Since $p \geqslant 2$ is arbitrary, the trajectories are a.s. Hölder continuous for any exponent less than 1 . We remark that there is no problem caused by the fact that we do not consider $\varepsilon=0$ (this is done by convenience of the proof); one may simply define $f(0)$ using the completeness of the space $L^{p}(\Omega)$.

Let us prove (3.3). We already noticed that it remains to identify the limit $f(0)=$ $\lim _{\varepsilon \rightarrow 0} f(\varepsilon)$. From the evolution equation (2.8) we can write 


$$
\begin{aligned}
& \int_{0}^{T} \int_{r}^{T} \frac{\partial p_{D}}{\partial y}(r, s, 0, \varepsilon) \varphi(s) \mathrm{d} s \mathrm{~d} V_{r} \\
& =\int_{0}^{T} \int_{r}^{T} \frac{\partial}{\partial y} q_{D}(r, s, 0, \varepsilon) \varphi(s) \mathrm{d} s \mathrm{~d} V_{r} \\
& \quad+\left.\sum_{j=1}^{n} \int_{0}^{T} \int_{r}^{T} \frac{\partial}{\partial y} \int_{r}^{s}\left(\int_{\mathbb{R}_{+}} q_{D}(u, s, z, \varepsilon) \frac{\partial p_{D}}{\partial z}(r, u, y, z) b_{j}(z) \mathrm{d} z\right) \mathrm{d} W_{u}^{j}\right|_{y=0} \mathrm{~d} s \mathrm{~d} V_{r} .
\end{aligned}
$$

The second term of the above identity, evaluated in $\varepsilon=0$, is zero. In order to finish the proof, it suffices to control that

$$
\left.\int_{0}^{\infty} \frac{\partial}{\partial y} q_{D}(0, s, y, 0)\right|_{y=0} \varphi(s+r) \mathrm{d} s=\varphi(r) .
$$

We get

$$
\left.\frac{\partial}{\partial y} q_{D}(0, s, y, 0)\right|_{y=0}=-\left.\frac{y}{s} q(0, s, y, 0)\right|_{y=0}
$$

and putting it in the above integral,

$$
\int_{0}^{\infty}-\left.\frac{y}{s} q(0, s, y, 0)\right|_{y=0} \varphi(s+r) \mathrm{d} s=\int_{0}^{\infty}-\left.\frac{y}{2 s \sqrt{\pi s}} \exp \left(-\frac{y^{2}}{4 s}\right) \varphi(s+r) \mathrm{d} s\right|_{y=0}
$$

by the change of variable $t=\sqrt{\frac{y^{2}}{4 s}}$ we read

$$
\int_{0}^{\infty}-\left.\frac{y}{s} q(0, s, y, 0)\right|_{y=0} \varphi(s+r) \mathrm{d} s=\frac{2}{\sqrt{\pi}} \int_{0}^{\infty} \exp \left(-t^{2}\right) \varphi(r) \mathrm{d} t=\varphi(r) .
$$

LEMMA 10. - For any $\alpha>0$ the following convergence holds for any $t \in[0, T]$, almost surely:

$$
x^{1+\alpha} \Psi(t, x) \rightarrow 0 \quad \text { as } x \rightarrow 0 .
$$

Proof. - The proof will follow if we show that for some $\alpha^{\prime}<\alpha$ the function $x^{1+\alpha^{\prime}} \Psi(t, x)$ is a.s. bounded in some neighborhood of 0 ; in turn, this is implied by a regularity result of this kind: there exists a version of the function $x^{1+\alpha^{\prime}} \Psi(t, x)$ that is almost surely Hölder continuous in an interval $[0, K], K>0$.

So we are lead to prove that for every $p \geqslant 2$ and $x, z \in(0, K), x<z$, the following estimate holds:

$$
\mathbb{E}\left|z^{1+\alpha} \Psi(t, z)-x^{1+\alpha} \Psi(t, x)\right|^{p} \leqslant C|z-x|^{p \alpha} .
$$

We can write

$$
\mathbb{E}\left|z^{1+\alpha} \Psi(t, z)-x^{1+\alpha} \Psi(t, x)\right|^{p} \leqslant A_{1}+A_{2},
$$


where

$$
\begin{aligned}
& A_{1}=x^{p(1+\alpha)} \mathbb{E}|\Psi(t, z)-\Psi(t, x)|^{p}, \\
& A_{2}=\left(z^{(1+\alpha)}-x^{(1+\alpha)}\right)^{p} \mathbb{E}|\Psi(t, z)|^{p} .
\end{aligned}
$$

In Eq. (3.2) we have proved that $\mathbb{E}|\Psi(t, z)|^{p} \leqslant C z^{-p}$ so that

$$
A_{2} \leqslant C|z-x|^{p \alpha} \text {. }
$$

On the other hand, we can estimate the difference

$$
\begin{aligned}
\mathbb{E}|\Psi(t, z)-\Psi(t, x)|^{p} & \leqslant C \mathbb{E}\left|\int_{0}^{t}\left[\frac{\partial p_{D}}{\partial y}(s, t, 0, z)-\frac{\partial p_{D}}{\partial y}(s, t, 0, x)\right]^{2} \mathrm{~d} s\right|^{p / 2} \\
& \leqslant C\left|\int_{0}^{t}\right|\left|\frac{\partial p_{D}}{\partial y}(s, t, 0, z)-\frac{\partial p_{D}}{\partial y}(s, t, 0, x) \|_{L^{p}(\Omega)}^{2} \mathrm{~d} s\right|^{p / 2} .
\end{aligned}
$$

By the estimates (2.10) and (2.11) we can easily deduce that (recall that we have chosen $x<z)$

$$
\begin{aligned}
\mathbb{E}|\Psi(t, z)-\Psi(t, x)|^{p} & \leqslant C|z-x|^{p \alpha}\left|\int_{0}^{t}(t-s)^{-2-\alpha} \exp \left(-\frac{x^{2}}{c(t-s)}\right) \mathrm{d} s\right|^{p / 2} \\
& \leqslant C|z-x|^{p \alpha} x^{-p(1+\alpha)}
\end{aligned}
$$

which proves the assertion in Eq. (3.8).

Now we are in the position to apply Kolmogorov's continuity theorem and the lemma is proved.

Remark 11. - The above proof shows in particular that $x \rightarrow \Psi(t, x)$ is a continuous function on $(0,+\infty)$ and that it is uniformly Hölder continuous on $(\delta,+\infty)$ for any $\delta>0$.

\section{Mild solution}

We recall from the introduction that $L_{\gamma}^{p}$ is the space of real-valued functions $f$ such that $\int_{0}^{\infty}|f(x)|^{p}\left(x^{p-1+\gamma} \wedge 1\right) \mathrm{d} x<\infty$, where $0<\gamma<1$ and $p \geqslant 2$. In order to prove Theorem 1 we need some preliminary results.

Lemma 12. - The application $\Lambda$ defined by

$$
(\Lambda \Phi)(t, x)=\sum_{j=1}^{n} \int_{0}^{t}\left(\int_{\mathbb{R}_{+}} p_{D}(s, t, y, x) \Phi(s, y) \mathrm{d} y\right) \mathrm{d} W_{s}^{j}
$$

is a contraction from $M^{p}\left(\Omega \times[0, T] ; L_{\gamma}^{p}\right)$ to $M^{p}\left(\Omega \times[0, T] ; L^{p}\left(\mathbb{R}_{+}\right)\right)$, for all $0<\gamma<1$ and $p \geqslant 2$. 
Proof. - Without loss of generality we can assume that $n=1$. We denote by $\mathcal{S}_{a}$ the space of simple and adapted processes of the form

$$
\phi(s)=\sum_{j=0}^{m-1} F_{j} \mathbf{1}_{\left(t_{j}, t_{j+1}\right]}(s),
$$

where $0<t_{0}<t_{1}<\cdots<t_{m}=T$ and the $F_{j}$ are $\mathcal{F}_{t_{j}}$-measurable random variables in $\mathcal{S}$.

Denote for simplicity

$$
B_{x}(s)=\int_{0}^{\infty} p_{D}(s, t, y, x) \Phi(s, y) \mathrm{d} y,
$$

so that $(\Lambda \Phi)(t, x)=\int_{0}^{t} B_{x}(s) \mathrm{d} W_{s}$. We can assume that $\Phi$ is a simple and adapted process; suppose first that $p_{D}(s, t, y, x)$ is an elementary backward-adapted process, so that $B_{x}(s)$ satisfies the assumption of Proposition 4; consider the family of functions

$$
F_{N}(x)=\int_{0}^{|x|} \int_{0}^{y}\left(p(p-1) z^{p-2} \wedge N\right) \mathrm{d} z \mathrm{~d} y ;
$$

by Itô's formula we obtain that

$$
\begin{aligned}
\mathbb{E}\left(F_{N}(\Lambda \Phi(t, x))\right)= & \mathbb{E} \int_{0}^{t} F_{N}^{\prime \prime}(\Lambda \Phi(s, x)) B_{x}^{2}(s) \mathrm{d} s \\
& +\frac{1}{2} \mathbb{E} \int_{0}^{t} F_{N}^{\prime \prime}(\Lambda \Phi(s, x)) B_{x}(s)\left(\int_{0}^{s} D_{s} B_{x}(r) \mathrm{d} W_{r}\right) \mathrm{d} s .
\end{aligned}
$$

By means of a density argument, we can easily check that Eq. (4.2) holds also for the kernel $p_{D}(s, t, y, x)$ introduced in the previous sections.

Now from the bound $F_{N}^{\prime \prime}(x) \leqslant p(p-1)\left(F_{N}(x)\right)^{(p-2) / p}$ it follows that

$$
\begin{aligned}
\mathbb{E}\left(F_{N}(\Lambda \Phi(t, x))\right) \leqslant & p(p-1) \int_{0}^{t}\left(\mathbb{E}\left|F_{N}(\Lambda \Phi(s, x))\right|\right)^{\frac{p-2}{p}}\left(\mathbb{E}\left|B_{x}(s)\right|^{p}\right)^{2 / p} \mathrm{~d} s \\
& +\frac{p(p-1)}{2} \int_{0}^{t}\left(\mathbb{E}\left|F_{N}(\Lambda \Phi(s, x))\right|\right)^{\frac{p-2}{p}} \\
& \times\left(\mathbb{E}\left|B_{x}(s)\left(\int_{0}^{s} D_{s} B_{x}(r), \mathrm{d} W_{r}\right)\right|^{p}\right)^{2 / p} \mathrm{~d} s .
\end{aligned}
$$

Applying the lemma on p. 171 of [17] we obtain that

$$
\mathbb{E}\left(F_{N}(\Lambda \Phi(t, x))\right) \leqslant c_{p}\left\{\int_{0}^{t}\left(\mathbb{E}\left|B_{x}(s)\right|^{p}\right)^{2 / p} \mathrm{~d} s\right.
$$




$$
\left.+\int_{0}^{t}\left(\mathbb{E}\left|B_{x}(s)\left(\int_{0}^{s} D_{s} B_{x}(r) \mathrm{d} W_{r}\right)\right|^{p / 2}\right)^{2 / p} \mathrm{~d} s\right\}^{p / 2} .
$$

This gives us, by Fatou's lemma, that

$$
\begin{aligned}
\mathbb{E}|\Lambda \Phi(t, x)|^{p} \leqslant & C\left\{\int_{0}^{t}\left(\mathbb{E}\left|B_{x}(s)\right|^{p}\right)^{2 / p} \mathrm{~d} s\right. \\
& \left.+\int_{0}^{t}\left(\mathbb{E}\left|B_{x}(s)\left(\int_{0}^{s} D_{s} B_{x}(r) \mathrm{d} W_{r}\right)\right|^{p / 2}\right)^{2 / p} \mathrm{~d} s\right\}^{p / 2}
\end{aligned}
$$

that we can write

$$
\|\Lambda \Phi(t, x)\|_{L^{p}(\Omega)}^{p} \leqslant C\left\{\phi_{1}(t, x)+\phi_{2}(t, x)\right\} .
$$

A few computations show that the first term can be decomposed

$$
\phi_{1}(t, x) \leqslant C_{1}\left\{\phi_{1,1}(t, x)+\phi_{1,2}(t, x)\right\},
$$

where

$$
\begin{aligned}
& \phi_{1,1}(t, x)=\left\{\int_{0}^{t}\left(\int_{0}^{1}\left\|p_{D}(s, t, y, x)\right\|_{L^{p}(\Omega)}\|\Phi(s, y)\|_{L^{p}(\Omega)} \mathrm{d} y\right)^{2} \mathrm{~d} s\right\}^{p / 2}, \\
& \phi_{1,2}(t, x)=\left\{\int_{0}^{t}\left(\int_{1}^{\infty}\left\|p_{D}(s, t, y, x)\right\|_{L^{p}(\Omega)}\|\Phi(s, y)\|_{L^{p}(\Omega)} \mathrm{d} y\right)^{2} \mathrm{~d} s\right\}^{p / 2} .
\end{aligned}
$$

We consider the second term since it is possible to bound the first one with the same estimates. We have

$$
\begin{aligned}
\phi_{2}(t, x)= & \left\{\int _ { 0 } ^ { t } \left[\mathbb { E } \left(\int_{\mathbb{R}_{+}} \int_{\mathbb{R}_{+}} p_{D}(s, t, x, y) \Phi(s, y)\right.\right.\right. \\
& \left.\left.\left.\times(\Lambda \Phi)(s, u)\left(D_{s}^{-} p_{D}(s, t, u, x)\right) \mathrm{d} u \mathrm{~d} y\right)^{p / 2}\right]^{2 / p} \mathrm{~d} s\right\}^{p / 2}
\end{aligned}
$$

proceeding as in the first case we get

$$
\phi_{2}(t, x) \leqslant C_{2}\left\{\phi_{2,1}(t, x)+\phi_{2,2}(t, x)+\phi_{3}(t, x)\right\},
$$

where we have defined, for some $q>0$,

$$
\begin{aligned}
& \phi_{2,1}(t, x)=\left\{\int_{0}^{t}(t-s)^{-q}\left(\int_{0}^{1}\left\|p_{D}(s, t, y, x)\right\|_{L^{p}(\Omega)}\|\Phi(s, y)\|_{L^{p}(\Omega)} \mathrm{d} y\right)^{2} \mathrm{~d} s\right\}^{p / 2}, \\
& \phi_{2,2}(t, x)=\left\{\int_{0}^{t}(t-s)^{-q}\left(\int_{1}^{\infty}\left\|p_{D}(s, t, y, x)\right\|_{L^{p}(\Omega)}\|\Phi(s, y)\|_{L^{p}(\Omega)} \mathrm{d} y\right)^{2} \mathrm{~d} s\right\}^{p / 2},
\end{aligned}
$$




$$
\phi_{3}(t, x)=\left\{\int_{0}^{t}(t-s)^{q}\left(\int_{\mathbb{R}_{+}}\left\|D_{s}^{-} p_{D}(s, t, u, x)\right\|_{L^{p}(\Omega)}\|(\Lambda \Phi)(s, u)\|_{L^{p}(\Omega)} \mathrm{d} u\right)^{2} \mathrm{~d} s\right\}^{p / 2} .
$$

Our next aim is to prove estimates for $\phi_{2,1}(t, x)$ and $\phi_{2,2}(t, x)$ (with the same techniques we will bound $\left.\phi_{1}(t, x)\right)$. Let us start from the first one: for some $a \in[0,1]$ and $b \in \mathbb{R}$ to be fixed later, we use Remark 7 to estimate

$$
\begin{aligned}
\phi_{2,1}(t, x) \leqslant & C_{2,1}\left\{\int_{0}^{t}(t-s)^{-q-1-a}\left(\int_{0}^{1} y^{a} \exp \left(-\frac{(y-x)^{2}}{c(t-s)}\right)\|\Phi(s, y)\|_{L^{p}(\Omega)} \mathrm{d} y\right)^{2} \mathrm{~d} s\right\}^{p / 2} \\
\leqslant & C_{2,1}\left\{\int_{0}^{t}(t-s)^{-q-1-a}\left(\int_{0}^{1} y^{2(b-1)} \exp \left(-\frac{(y-x)^{2}}{c(t-s)}\right) \mathrm{d} y\right)\right. \\
& \left.\times\left(\int_{0}^{1} y^{2(a-b+1)} \exp \left(-\frac{(y-x)^{2}}{c(t-s)}\right)\|\Phi(s, y)\|_{L^{p}(\Omega)}^{2} \mathrm{~d} y\right) \mathrm{d} s\right\}^{p / 2} .
\end{aligned}
$$

We recall the following estimate, which holds for any $b>1 / 2$ :

$$
\int_{0}^{1} y^{2(b-1)} \exp \left(-\frac{(y-x)^{2}}{c(t-s)}\right) d y \leqslant C
$$

so that

$$
\begin{aligned}
\phi_{2,1}(t, x) \leqslant & C_{2,1}\left\{\int_{0}^{t}(t-s)^{-q-1-a}\right. \\
& \left.\times\left(\int_{0}^{1} y^{2(a-b+1)} \exp \left(-\frac{(y-x)^{2}}{c(t-s)}\right)\|\Phi(s, y)\|_{L^{p}(\Omega)}^{2} \mathrm{~d} y\right) \mathrm{d} s\right\}^{p / 2} .
\end{aligned}
$$

We integrate in $\mathrm{d} x$ :

$$
\begin{aligned}
\int_{\mathbb{R}_{+}} \phi_{2,1}(t, x) \mathrm{d} x \leqslant & C_{2,1} \int_{\mathbb{R}_{+}}\left\{\int_{0}^{t}(t-s)^{-q-1-a}\right. \\
& \left.\times\left(\int_{0}^{1} y^{2(a-b+1)} \exp \left(-\frac{(y-x)^{2}}{c(t-s)}\right)\|\Phi(s, y)\|_{L^{p}(\Omega)}^{2} \mathrm{~d} y\right) \mathrm{d} s\right\}^{p / 2} \mathrm{~d} x
\end{aligned}
$$

and by Hölder's inequality

$$
\begin{aligned}
\int_{\mathbb{R}_{+}} \phi_{2,1}(t, x) \mathrm{d} x \leqslant & C_{2,1} \int_{\mathbb{R}_{+}}\left\{\int_{0}^{t}(t-s)^{-q-1-a+\frac{1}{2}-\frac{1}{p}}\right. \\
& \left.\times\left(\int_{0}^{1} y^{p(a-b+1)} \exp \left(-\frac{(y-x)^{2}}{c(t-s)}\right)\|\Phi(s, y)\|_{L^{p}(\Omega)}^{p} \mathrm{~d} y\right)^{2 / p} \mathrm{~d} s\right\}^{p / 2} \mathrm{~d} x .
\end{aligned}
$$


The desired estimate follows by applying Minkowski's and Hölder's inequalities, under the assumption $q+a<1 / 2$ :

$$
\int_{\mathbb{R}_{+}} \phi_{2,1}(t, x) \mathrm{d} x \leqslant C_{2,1} \int_{0}^{t}(t-s)^{-q-\frac{1}{2}-a} \int_{0}^{1} y^{p(a-b+1)}\|\Phi(s, y)\|_{L^{p}(\Omega)}^{p} \mathrm{~d} y \mathrm{~d} s .
$$

We consider now the second term. Assume that $q<1$ :

$$
\begin{aligned}
\phi_{2,2}(t, x) \leqslant & C_{2,2} t^{(1-q)(p / 2-1)} \int_{0}^{t}(t-s)^{-q} \\
& \times\left(\int_{1}^{\infty}\left\|p_{D}(s, t, y, x)\right\|_{L^{p}(\Omega)}\|\Phi(s, y)\|_{L^{p}(\Omega)} \mathrm{d} y\right)^{p} \mathrm{~d} s .
\end{aligned}
$$

We integrate both sides of the above inequality with respect to $\mathrm{d} x$; using estimate (2.10) and Hölder's inequality we get

$$
\int_{\mathbb{R}_{+}} \phi_{2,2}(t, x) \mathrm{d} x \leqslant C_{2,2} t^{(1-q)\left(\frac{p}{2}-1\right)} \int_{0}^{t}(t-s)^{-q} \int_{1}^{\infty}\|\Phi(s, y)\|_{L^{p}(\Omega)}^{p} \mathrm{~d} y \mathrm{~d} s .
$$

We need to take some care in order to evaluate $\phi_{3}(t, x)$. Using (2.14) and Schwartz's inequality we may write

$$
\begin{aligned}
\phi_{3}(t, x) \leqslant & C_{3}\left\{\int _ { 0 } ^ { t } ( t - s ) ^ { q } \left(\int_{\mathbb{R}_{+}} \exp \left(-\frac{(u-x)^{2}}{c(t-s)}\right)(t-s)^{-1}\right.\right. \\
& \times \\
& \left.\left.\|(\Lambda \Phi)(s, u)\|_{L^{p}(\Omega)} \mathrm{d} u\right)^{2} \mathrm{~d} s\right\}^{p / 2} \\
\leqslant & C_{3}\left\{\int_{0}^{t}(t-s)^{q-2}\left(\int_{\mathbb{R}_{+}} \exp \left(-\frac{(u-x)^{2}}{c(t-s)}\right) \mathrm{d} u\right)\right. \\
& \left.\times\left(\int_{\mathbb{R}_{+}} \exp \left(-\frac{(u-x)^{2}}{c(t-s)}\right)\|(\Lambda \Phi)(s, u)\|_{L^{p}(\Omega)}^{2} \mathrm{~d} u\right) \mathrm{d} s\right\}^{p / 2} \\
\leqslant & C_{3}\left\{\int_{0}^{t}(t-s)^{q-\frac{3}{2}} \int_{\mathbb{R}_{+}} \exp \left(-\frac{(u-x)^{2}}{c(t-s)}\right)\|(\Lambda \Phi)(s, u)\|_{L^{p}(\Omega)}^{2} \mathrm{~d} u \mathrm{~d} s\right\}^{p / 2} .
\end{aligned}
$$

The integration in $\mathrm{d} x$ leads to

$$
\int_{\mathbb{R}_{+}} \phi_{3}(t, x) \mathrm{d} x
$$




$$
\leqslant C_{3} \iint_{\mathbb{R}_{+}}\left\{\int_{0}^{t}(t-s)^{q-3 / 2} \int_{\mathbb{R}_{+}} \exp \left(-\frac{(u-x)^{2}}{c(t-s)}\right)\|(\Lambda \Phi)(s, u)\|_{L^{p}(\Omega)}^{2} \mathrm{~d} u \mathrm{~d} s\right\}^{p / 2} \mathrm{~d} x
$$

we apply first Hölder's inequality

$$
\begin{aligned}
\int_{\mathbb{R}_{+}} \phi_{3}(t, x) \mathrm{d} x \leqslant & C_{3} \int_{\mathbb{R}_{+}}\left\{\int_{0}^{t}(t-s)^{q-1-\frac{1}{p}}\right. \\
& \left.\times\left(\int_{\mathbb{R}_{+}} \exp \left(-\frac{(u-x)^{2}}{c(t-s)}\right)\|(\Lambda \Phi)(s, u)\|_{L^{p}(\Omega)}^{p} \mathrm{~d} u\right)^{2 / p} \mathrm{~d} s\right\}^{p / 2} \mathrm{~d} x
\end{aligned}
$$

and then Minkowski's inequality

$$
\int_{\mathbb{R}_{+}} \phi_{3}(t, x) \mathrm{d} x \leqslant C_{3}\left\{\int_{0}^{t}(t-s)^{q-1}\left(\int_{\mathbb{R}_{+}}\|(\Lambda \Phi)(s, u)\|_{L^{p}(\Omega)}^{p} \mathrm{~d} u\right)^{2 / p} \mathrm{~d} s\right\}^{p / 2} .
$$

By another application of Hölder's inequality we have

$$
\int_{\mathbb{R}_{+}} \phi_{3}(t, x) \mathrm{d} x \leqslant C_{3} \int_{0}^{t}(t-s)^{q-1} \int_{\mathbb{R}_{+}}\|(\Lambda \Phi)(s, u)\|_{L^{p}(\Omega)}^{p} \mathrm{~d} u \mathrm{~d} s .
$$

We now define $\delta=\frac{1}{2}-a>q$ and $b-a=(1-\gamma) / p$. So far we have proved the following estimate

$$
\begin{aligned}
\int_{\mathbb{R}_{+}}\|\Lambda \Phi(t, x)\|_{L^{p}(\Omega)}^{p} \mathrm{~d} x \leqslant & C_{1,1} \int_{0}^{t}(t-s)^{\delta-1} \int_{0}^{1} x^{p-1+\gamma}\|\Phi(s, x)\|_{L^{p}(\Omega)}^{p} \mathrm{~d} x \mathrm{~d} s \\
& +C_{2,1} \int_{0}^{t}(t-s)^{\delta-q-1} \int_{0}^{1} x^{p-1+\gamma}\|\Phi(s, x)\|_{L^{p}(\Omega)}^{p} \mathrm{~d} x \mathrm{~d} s \\
& +C_{1,2} \int_{0}^{t} \int_{1}^{\infty}\|\Phi(s, x)\|_{L^{p}(\Omega)}^{p} \mathrm{~d} x \mathrm{~d} s \\
& +C_{2,2} \int_{0}^{t}(t-s)^{-q} \int_{1}^{\infty}\|\Phi(s, x)\|_{L^{p}(\Omega)}^{p} \mathrm{~d} x \mathrm{~d} s \\
& +C_{3} \int_{0}^{t}(t-s)^{q-1} \int_{\mathbb{R}_{+}}\|(\Lambda \Phi)(s, x)\|_{L^{p}(\Omega)}^{p} \mathrm{~d} x \mathrm{~d} s .
\end{aligned}
$$

A simple Gronwall-like argument shows that the above estimate becomes 


$$
\begin{aligned}
\int_{\mathbb{R}_{+}}\|(\Lambda \Phi)(t, x)\|_{L^{p}(\Omega)}^{p} \mathrm{~d} x \leqslant & C_{1,1} \int_{0}^{t}(t-s)^{\delta-1} \int_{0}^{1} x^{p-1+\gamma}\|\Phi(s, x)\|_{L^{p}(\Omega)}^{p} \mathrm{~d} x \mathrm{~d} s \\
& +C_{2,1} \int_{0}^{t}(t-s)^{\delta-q-1} \int_{0}^{1} x^{p-1+\gamma}\|\Phi(s, x)\|_{L^{p}(\Omega)}^{p} \mathrm{~d} x \mathrm{~d} s \\
& +C_{1,2} \int_{0}^{t} \int_{1}^{\infty}\|\Phi(s, x)\|_{L^{p}(\Omega)}^{p} \mathrm{~d} x \mathrm{~d} s \\
& +C_{2,2} \int_{0}^{t}(t-s)^{-q} \int_{1}^{\infty}\|\Phi(s, x)\|_{L^{p}(\Omega)}^{p} \mathrm{~d} x \mathrm{~d} s .
\end{aligned}
$$

Now we are able to integrate between 0 and $T$; repeated applications of Fubini's theorem imply

$$
\begin{aligned}
& \int_{0}^{T} \int_{\mathbb{R}_{+}}\|(\Lambda \Phi)(t, x)\|_{L^{p}(\Omega)}^{p} \mathrm{~d} x \mathrm{~d} t \\
& \leqslant\left(C_{1,1} T^{\delta}+C_{2,1} T^{\delta-q}\right) \int_{0}^{T} \int_{0}^{1} x^{p-1+\gamma}\|\Phi(s, x)\|_{L^{p}(\Omega)}^{p} \mathrm{~d} x \mathrm{~d} s \\
& \quad+\left(C_{1,2} T+C_{2,2} T^{q}\right) \int_{0}^{T} \int_{1}^{\infty}\|\Phi(s, x)\|_{L^{p}(\Omega)}^{p} \mathrm{~d} x \mathrm{~d} s \\
& \leqslant C T \int_{0}^{T} \int_{0}^{\infty}\left(1 \wedge x^{p-1+\gamma}\right)\|\Phi(s, x)\|_{L^{p}(\Omega)}^{p} \mathrm{~d} x \mathrm{~d} s,
\end{aligned}
$$

so for every positive time $T$ such that $C T<1$ we have proven that $\Lambda: L^{p}(\Omega \times$ $\left.[0, T] ; L_{\gamma}^{p}\right) \rightarrow L^{p}\left(\Omega \times[0, T] ; L^{p}\left(\mathbb{R}_{+}\right)\right)$is a contraction. The restriction on $T$ can be easily avoided repeating the above arguments in $[T, 2 T]$, and so on.

In order to complete the proof we only need to control that all the parameters are well defined. Since for any $p \geqslant 2, \gamma \in(0,1)$ the interval $(0,(1-\gamma) / p)$ is non empty, it is sufficient to take $q$ inside this interval, which implies in particular that $\frac{1}{2}-q>\frac{\gamma}{p}+\frac{1}{2}-\frac{1}{p}>0$; take now $a \in\left(\frac{\gamma}{p}+\frac{1}{2}-\frac{1}{p}, \frac{1}{2}-q\right)$ : it is well defined and $b=a+(1-\gamma) / p$ verifies $b>1 / 2$. Now the proof is complete.

LEMMA 13. - Assume that $\Phi \in M^{p}\left(\Omega \times[0, T] ; L_{\gamma}^{p}\right)$ for some $p>2$ and $0<\gamma<1$. Then for any $t \in[0, T]$ and $a>(1+\gamma) / p$ the mapping

$$
x \longrightarrow x^{a}(\Lambda \Phi)(t, x)
$$

is almost surely uniformly Hölder continuous on $[0, K]$, for all $K>0$, of order $\rho$, where

$$
\rho<\frac{1}{2}\left(a-\frac{1+\gamma}{p}\right) \wedge\left(\frac{1}{2}-\frac{1}{p}\right) .
$$


Proof. - We try to apply the same construction as in the previous lemma. Let us denote

$$
B_{x, z}(s)=\int_{0}^{\infty}\left[p_{D}(s, t, y, x)-p_{D}(s, t, y, z)\right] \Phi(s, y) \mathrm{d} y,
$$

and $X(t, x, z)=\int_{0}^{t} B_{x, z}(s) \mathrm{d} W_{s}$. A few calculations show that

$$
\begin{aligned}
& \mathbb{E}|X(t, x, z)|^{p} \\
& \leqslant C\left\{\int_{0}^{t}\left(\mathbb{E}\left|B_{x, z}(s)\right|^{p}\right)^{2 / p} \mathrm{~d} s+\int_{0}^{t}\left(\mathbb{E}\left|B_{x, z}(s)\left(\int_{0}^{s} D_{s} B_{x, z}(r) \mathrm{d} W_{r}\right)\right|^{p / 2}\right)^{2 / p} \mathrm{~d} s\right\}^{p / 2} \\
& \leqslant C\left|\int_{0}^{t}\left\|B_{x, z}(s)\right\|_{L^{p}(\Omega)}^{2} \mathrm{~d} s\right|^{p / 2}+C\left|\int_{0}^{t}\left\|B_{x, z}(s)\left(\int_{0}^{s} D_{s} B_{x, z}(r) \mathrm{d} W_{r}\right)\right\|_{L^{p / 2}(\Omega)} \mathrm{d} s\right|^{p / 2} \\
& =I_{1}+I_{2} .
\end{aligned}
$$

We are interested mainly in the second term. Let $q$ be a parameter in $(0,1)$ that will be chosen later.

$$
\begin{aligned}
I_{2} \leqslant & \mid \int_{0}^{t}(t-s)^{-q}\left(\int_{\mathbb{R}_{+}}\left\|p_{D}(s, t, y, x)-p_{D}(s, t, y, z)\right\|_{L^{p}(\Omega)}\|\Phi(s, y)\|_{L^{p}(\Omega)} \mathrm{d} y\right)^{2} \mathrm{~d} s \\
& +\int_{0}^{t}(t-s)^{q}\left(\int_{\mathbb{R}_{+}}\left\|D_{s}^{-} p_{D}(s, t, y, x)-D_{s}^{-} p_{D}(s, t, y, z)\right\|_{L^{p}(\Omega)}\right. \\
& \left.\times\|\Lambda \Phi(s, y)\|_{L^{p}(\Omega)} \mathrm{d} y\right)\left.^{2} \mathrm{~d} s\right|^{p / 2} .
\end{aligned}
$$

Let us write

$$
\begin{aligned}
I_{2,1}= & \int_{0}^{t}(t-s)^{-q}\left(\int_{\mathbb{R}_{+}}\left\|p_{D}(s, t, y, x)-p_{D}(s, t, y, z)\right\|_{L^{p}(\Omega)}\|\Phi(s, y)\|_{L^{p}(\Omega)} \mathrm{d} y\right)^{2} \mathrm{~d} s, \\
I_{2,2}= & \int_{0}^{t}(t-s)^{q} \\
& \times\left(\int_{\mathbb{R}_{+}}\left\|D_{s}^{-} p_{D}(s, t, y, x)-D_{s}^{-} p_{D}(s, t, y, z)\right\|_{L^{p}(\Omega)}\|\Lambda \Phi(s, y)\|_{L^{p}(\Omega)} \mathrm{d} y\right)^{2} \mathrm{~d} s .
\end{aligned}
$$

In order to estimate the first term we notice that for all constants $c \in(0,1)$ and $d>0$ :

$$
\begin{aligned}
& \left\|p_{D}(s, t, y, x)-p_{D}(s, t, y, z)\right\|_{L^{p}(\Omega)} \\
& \quad \leqslant C(t-s)^{-\frac{1}{2}-\frac{c}{2}-\frac{d}{2}}|x-z|^{c} y^{d}\left[\exp \left(-\frac{(y-x)^{2}}{c(t-s)}\right)+\exp \left(-\frac{(y-z)^{2}}{c(t-s)}\right)\right]
\end{aligned}
$$


from where it comes that

$$
\begin{aligned}
I_{2,1} \leqslant & C|x-z|^{2 c} \int_{0}^{t}(t-s)^{-q-1-c-d} \\
& \times\left(\int_{[0,1]} y^{d}\left[\exp \left(-\frac{(y-x)^{2}}{c(t-s)}\right)+\exp \left(-\frac{(y-z)^{2}}{c(t-s)}\right)\right]\|\Phi(s, y)\|_{L^{p}(\Omega)} \mathrm{d} y\right)^{2} \mathrm{~d} s \\
& +C|x-z|^{2 c} \int_{0}^{t}(t-s)^{-q-1-c} \\
& \times\left(\int_{[1, \infty)}\left[\exp \left(-\frac{(y-x)^{2}}{c(t-s)}\right)+\exp \left(-\frac{(y-z)^{2}}{c(t-s)}\right)\right]\|\Phi(s, y)\|_{L^{p}(\Omega)} \mathrm{d} y\right)^{2} \mathrm{~d} s .
\end{aligned}
$$

We now use the following estimate, where $\delta>\frac{1}{2}$ and $a>0$ :

$$
\int_{0}^{1} y^{2(\delta-1)} \exp \left(-\frac{(y-x)^{2}}{c(t-s)}\right) \mathrm{d} y \leqslant C(t-s)^{a+\delta-1 / 2} x^{-2 a}
$$

and we get

$$
\begin{aligned}
& \left(\int_{0}^{1} y^{d}\left[\exp \left(-\frac{(y-x)^{2}}{c(t-s)}\right)+\exp \left(-\frac{(y-z)^{2}}{c(t-s)}\right)\right]\|\Phi(s, y)\|_{L^{p}(\Omega)} \mathrm{d} y\right)^{2} \\
& \leqslant C(t-s)^{a+\delta-\frac{1}{2}} \int_{[0,1]} y^{2(d+1-\delta)} \\
& \quad \times\left[\exp \left(-\frac{(y-x)^{2}}{c(t-s)}\right) x^{-2 a}+\exp \left(-\frac{(y-z)^{2}}{c(t-s)}\right) z^{-2 a}\right]\|\Phi(s, y)\|_{L^{p}(\Omega)}^{2} \mathrm{~d} y
\end{aligned}
$$

so that

$$
\begin{aligned}
I_{2,1} \leqslant & C|x-z|^{2 c} \int_{0}^{t}(t-s)^{-q-1-c-d+a+\delta-\frac{1}{2}}\left(\int_{0}^{1} y^{2(d+1-\delta)}\right. \\
& \left.\times\left[\exp \left(-\frac{(y-x)^{2}}{c(t-s)}\right) x^{-2 a}+\exp \left(-\frac{(y-z)^{2}}{c(t-s)}\right) z^{-2 a}\right]\|\Phi(s, y)\|_{L^{p}(\Omega)}^{2} \mathrm{~d} y\right) \mathrm{d} s \\
& +C|x-z|^{2 c} \int_{0}^{t}(t-s)^{-q-\frac{1}{2}-c} \\
& \times\left(\int_{1}^{\infty}\left[\exp \left(-\frac{(y-x)^{2}}{c(t-s)}\right)+\exp \left(-\frac{(y-z)^{2}}{c(t-s)}\right)\right]\|\Phi(s, y)\|_{L^{p}(\Omega)}^{2} \mathrm{~d} y\right) \mathrm{d} s .
\end{aligned}
$$

Taking $x, z \in[0, K]$ we can write 


$$
\begin{aligned}
I_{2,1} \leqslant & C|x-z|^{2 c} \int_{0}^{t}(t-s)^{-q-1 / 2-c+[(a+\delta-1-d) \wedge 0]}\left(\int_{\mathbb{R}_{+}}\left(y^{2(d+1-\delta)} \wedge 1\right)\right. \\
& \left.\times\left[\exp \left(-\frac{(y-x)^{2}}{c(t-s)}\right) x^{-2 a}+\exp \left(-\frac{(y-z)^{2}}{c(t-s)}\right) z^{-2 a}\right]\|\Phi(s, y)\|_{L^{p}(\Omega)}^{2} \mathrm{~d} y\right) \mathrm{d} s .
\end{aligned}
$$

Notice that, by Hölder's inequality

$$
\begin{aligned}
& \int_{\mathbb{R}_{+}}\left(y^{2(d+1-\delta)} \wedge 1\right)\left[\exp \left(-\frac{(y-x)^{2}}{c(t-s)}\right) x^{-2 a}+\exp \left(-\frac{(y-z)^{2}}{c(t-s)}\right) z^{-2 a}\right]\|\Phi(s, y)\|_{L^{p}(\Omega)}^{2} \mathrm{~d} y \\
& \leqslant\left(\int_{\mathbb{R}_{+}}\left(y^{p(d+1-\delta)} \wedge 1\right)\left[\exp \left(-\frac{(y-x)^{2}}{c(t-s)}\right) x^{-p a}+\exp \left(-\frac{(y-z)^{2}}{c(t-s)}\right) z^{-p a}\right]\right. \\
& \left.\quad \times\|\Phi(s, y)\|_{L^{p}(\Omega)}^{p} \mathrm{~d} y\right)^{2 / p}\left(\int_{\mathbb{R}_{+}}\left[\exp \left(-\frac{(y-x)^{2}}{c(t-s)}\right)+\exp \left(-\frac{(y-z)^{2}}{c(t-s)}\right)\right] \mathrm{d} y\right)^{\frac{p-2}{p}},
\end{aligned}
$$

from where it follows that

$$
\begin{aligned}
I_{2,1} \leqslant & C|x-z|^{2 c} \int_{0}^{t}(t-s)^{-q-1 / p-c+[(a+\delta-1-d) \wedge 0]}\left(\int_{\mathbb{R}_{+}}\left(y^{p(d+1-\delta)} \wedge 1\right)\right. \\
& \left.\times\left[\exp \left(-\frac{(y-x)^{2}}{c(t-s)}\right) x^{-p a}+\exp \left(-\frac{(y-z)^{2}}{c(t-s)}\right) z^{-p a}\right]\|\Phi(s, y)\|_{L^{p}(\Omega)}^{p} \mathrm{~d} y\right)^{2 / p} \mathrm{~d} s .
\end{aligned}
$$

Notice that $I_{1} \leqslant\left|I_{2,1}\right|^{p / 2}$ since we can choose $q=0$ in the definition. Hence this term also is bounded by $I_{2}$.

Now we consider the second term. Applying Hölder's inequality we get

$$
\begin{aligned}
I_{2,2} \leqslant & C|x-z|^{2 c} \int_{0}^{t}(t-s)^{q-2-c} \\
& \times\left(\int_{\mathbb{R}_{+}}\left[\exp \left(-\frac{(y-x)^{2}}{c(t-s)}\right)+\exp \left(-\frac{(y-z)^{2}}{c(t-s)}\right)\right]\|\Lambda \Phi(s, y)\|_{L^{p}(\Omega)} \mathrm{d} y\right)^{2} \mathrm{~d} s \\
\leqslant & C|x-z|^{2 c} \int_{0}^{t}(t-s)^{q-\frac{3}{2}-c} \\
& \times\left(\int_{\mathbb{R}_{+}}\left[\exp \left(-\frac{(y-x)^{2}}{c(t-s)}\right)+\exp \left(-\frac{(y-z)^{2}}{c(t-s)}\right)\right]\|\Lambda \Phi(s, y)\|_{L^{p}(\Omega)}^{2} \mathrm{~d} y\right) \mathrm{d} s \\
\leqslant & C|x-z|^{2 c} \int_{0}^{t}(t-s)^{q-1-c-\frac{1}{p}}
\end{aligned}
$$




$$
\times\left(\int_{\mathbb{R}_{+}}\left[\exp \left(-\frac{(y-x)^{2}}{c(t-s)}\right)+\exp \left(-\frac{(y-z)^{2}}{c(t-s)}\right)\right]\|\Lambda \Phi(s, y)\|_{L^{p}(\Omega)}^{p} \mathrm{~d} y\right)^{2 / p} \mathrm{~d} s .
$$

In order to apply Garsia-Rodemich-Rumsay's lemma we need to estimate the quantity

$$
R:=\int_{0}^{K} \int_{0}^{K} \frac{\left\|x^{a}(\Lambda \Phi)(t, x)-z^{a}(\Lambda \Phi)(t, z)\right\|_{L^{p}(\Omega)}^{p}}{|x-z|^{m+2}} \mathrm{~d} z \mathrm{~d} x
$$

for a parameter $m$ such that $0<m<p c-1$. The symmetry of the problem yields

$$
\begin{aligned}
R & =2 \int_{0}^{K} \int_{0}^{z} \frac{\left\|x^{a}(\Lambda \Phi)(t, x)-z^{a}(\Lambda \Phi)(t, z)\right\|_{L^{p}(\Omega)}^{p}}{|x-z|^{m+2}} \mathrm{~d} x \mathrm{~d} z \\
\leqslant & C \int_{0}^{K} \int_{0}^{z}|x-z|^{p c-m-2} \mid \int_{0}^{t}(t-s)^{q-1-c-\frac{1}{p}} \\
& \times\left.\left(\int_{\mathbb{R}_{+}}\left[\exp \left(-\frac{(y-x)^{2}}{c(t-s)}\right)+\exp \left(-\frac{(y-z)^{2}}{c(t-s)}\right)\right]\|\Lambda \Phi(s, y)\|_{L^{p}(\Omega)}^{p} \mathrm{~d} y\right)^{2 / p} \mathrm{~d} s\right|^{p / 2} \mathrm{~d} x \mathrm{~d} z \\
& +C \int_{0}^{K} \int_{0}^{z}|x-z|^{p c-m-2} \mid \int_{0}^{t}(t-s)^{-q-c-1 p+[(a+\delta-d-1) \wedge 0]}\left(\int_{\mathbb{R}_{+}}\left(y^{p(d+1-\delta)} \wedge 1\right)\right. \\
& \left.\times\left[\exp \left(-\frac{(y-x)^{2}}{c(t-s)}\right)+\exp \left(-\frac{(y-z)^{2}}{c(t-s)}\right)\right]\|\Phi(s, y)\|_{L^{p}(\Omega)}^{p} \mathrm{~d} y\right)\left.^{2 / p} \mathrm{~d} s\right|^{p / 2} \mathrm{~d} x \mathrm{~d} z \\
& +C \int_{0}^{K} \int_{0}^{z}\left|x^{a}-z^{a}\right|^{p}|x-z|^{p c-m-2}\|\Lambda \Phi(t, z)\|_{L^{p}(\Omega)}^{p} \mathrm{~d} x \mathrm{~d} z
\end{aligned}
$$

that is,

$$
\begin{aligned}
R \leqslant & \left.C \int_{0}^{K} \int_{0}^{t}(t-s)^{q-1-c-\frac{1}{p}}\left(\int_{\mathbb{R}_{+}} \exp \left(-\frac{(y-z)^{2}}{c(t-s)}\right)\|\Lambda \Phi(s, y)\|_{L^{p}(\Omega)}^{p} \mathrm{~d} y\right)^{2 / p} \mathrm{~d} s\right|^{p / 2} \mathrm{~d} z \\
& +C \int_{0}^{K} \mid \int_{0}^{t}(t-s)^{-q-c-1 / p+[(a+\delta-d-1) \wedge 0]} \\
& \times\left.\left(\int_{\mathbb{R}_{+}}\left(y^{p(d+1-\delta)} \wedge 1\right) \exp \left(-\frac{(y-z)^{2}}{c(t-s)}\right)\|\Phi(s, y)\|_{L^{p}(\Omega)}^{p} \mathrm{~d} y\right)^{2 / p} \mathrm{~d} s\right|^{p / 2} \mathrm{~d} z \\
& +C \int_{0}^{K}\|\Lambda \Phi(t, z)\|_{L^{p}(\Omega)}^{p} \mathrm{~d} z
\end{aligned}
$$


and finally

$$
\begin{aligned}
R \leqslant & C \int_{0}^{K}\left|\int_{0}^{t}(t-s)^{q-1-c-\frac{1}{p}}\left(\int_{\mathbb{R}_{+}}\|\Lambda \Phi(s, y)\|_{L^{p}(\Omega)}^{p} \mathrm{~d} y\right)^{2 / p} \mathrm{~d} s\right|^{p / 2} \\
+ & C \mid \int_{0}^{t}(t-s)^{-q-c-1 / p+[(a+\delta-d-1) \wedge 0]} \\
& \times\left.\left(\int_{\mathbb{R}_{+}}\left(y^{p(d+1-\delta)} \wedge 1\right)\|\Phi(s, y)\|_{L^{p}(\Omega)}^{p} \mathrm{~d} y\right)^{2 / p} \mathrm{~d} s\right|^{p / 2} .
\end{aligned}
$$

In order to complete the proof we only need to control that all the parameters are well defined. Actually, it is sufficient to take

$$
\begin{array}{r}
a>\frac{1+\gamma}{p}, \quad c \in\left(\frac{1}{p},\left(\frac{1-\gamma}{2 p}+\frac{a}{2}\right) \wedge \frac{1}{2}\right), \\
m \in(0, p c-1) \quad q \in\left(c, \frac{1-\gamma}{p}-c+a\right), \\
d>\frac{\gamma}{p}+\frac{1}{2}-\frac{1}{p}, \quad \delta=d+(1-\gamma) / p>1 / 2 .
\end{array}
$$

With this set of parameters, the right hand side of the above estimate is finite; from the Garsia-Rodemich-Rumsay's lemma it follows that $(\Lambda \Phi)(t, \cdot)$ is uniformly Hölder continuous with arbitrary parameter less than $\frac{1}{2}\left(a-\frac{1+\gamma}{p}\right) \wedge\left(\frac{1}{2}-\frac{1}{p}\right)$. The proof is now complete.

Proof of Theorem 1. - The proof of existence and uniqueness of the solution is based on the classical fixed point theorem for contractions. Let $\mathcal{K}$ be the following transformation

$$
(\mathcal{K} \phi)(t, x)=\Psi(t, x)+\sum_{j=1}^{n} \int_{0}^{t} \int_{\mathbb{R}_{+}} p_{D}(s, t, y, x) F_{j}(s, y, \phi(s, y)) \mathrm{d} y \mathrm{~d} W_{s}^{j} .
$$

We show first that for any $\lambda \in(0,1)$ the application $\mathcal{K}$ maps $M^{p}\left(\Omega \times[0, T] ; L_{\lambda}^{p}\right)$ into each $M^{p}\left(\Omega \times[0, T] ; L_{\gamma}^{p}\right)$. The boundary term $\Psi(t, x)$ belongs to $M^{p}\left(\Omega \times[0, T] ; L_{\gamma}^{p}\right)$ as it is shown in Section 3. In view of Lemma 12, it remains to prove that for any $\phi \in M^{p}\left(\Omega \times[0, T] ; L_{\lambda}^{p}\right)$ the process $\Phi(s, y)=F(s, y, \phi(s, y))$ belongs to $M^{p}(\Omega \times$ $\left.[0, T] ; L_{\lambda}^{p}\right)$. But we have

$$
\begin{aligned}
& \mathbb{E} \sum_{j=1}^{n} \int_{0}^{T} \int_{\mathbb{R}_{+}}\left|F_{j}(s, y, \phi(s, y))\right|^{p}\left(1 \wedge y^{p-1+\lambda}\right) \mathrm{d} y \mathrm{~d} s \\
& \quad \leqslant C \mathbb{E} \int_{0}^{T} \int_{\mathbb{R}_{+}}\left(|\phi(s, y)|^{p}+|h(y)|^{p}\right)\left(1 \wedge y^{p-1+\lambda}\right) \mathrm{d} y \mathrm{~d} s .
\end{aligned}
$$


Finally note that $\Lambda \Phi(s, y)$ belongs to $M^{p}\left(\Omega \times[0, T] ; L^{p}\left(\mathbb{R}_{+}\right)\right.$) (see the proof of Lemma 12) and $L^{p}\left(\mathbb{R}_{+}\right) \subset L_{\gamma}^{p}$ for any $\gamma \in(0,1)$.

Our next aim is to prove the boundary regularity result stated in Eq. (1.5). We have

$$
x^{1+\alpha} \Psi(t, x)+x^{1+\alpha} \sum_{j=1}^{n} \int_{0}^{t} \int_{\mathbb{R}_{+}} p_{D}(s, t, y, x) F_{j}(s, y, \phi(s, y)) \mathrm{d} y \mathrm{~d} W_{s}^{j} \rightarrow 0
$$

since the first term tends to 0 , as it is proved in Lemma 10, and the second one does the same, as a consequence of Lemma 13. Finally, the regularity of the solution is again a consequence of the proofs of Lemmas 10 and 13.

\section{Weak solution}

We devote this section to the proof of Theorem 3. The idea is to prove that the left hand side of Eq. (1.7) is equal to the term

$$
\int_{0}^{\infty}\left(\int_{r}^{\infty} \frac{\partial p_{D}}{\partial y}(r, s, 0, \varepsilon) \varphi(s) \mathrm{d} s\right) \mathrm{d} V_{r}
$$

which appears in Lemma 9 (where $\varphi(s)=\frac{\partial f}{\partial x}(s, 0)$ has compact support). For simplicity of notation we take $n=1$. Let us denote

$$
\begin{aligned}
\Psi(s, x) & =\int_{0}^{s} \frac{\partial p_{D}}{\partial y}(r, s, 0, x) \mathrm{d} V_{r}, \\
\Gamma(s, x) & =\int_{0}^{s} \int_{\mathbb{R}_{+}} p_{D}(r, s, y, x) F(r, y, u(r, y)) \mathrm{d} y \mathrm{~d} W_{r} .
\end{aligned}
$$

Fix a test function $f(t, x)$. We claim that

$$
\begin{aligned}
\int_{0}^{+\infty} \int_{\mathbb{R}_{+}} \Psi(s, x+\varepsilon)\left(\frac{\partial f}{\partial s}(s, x)+\frac{\partial^{2} f}{\partial x^{2}}(s, x)\right) \mathrm{d} x \mathrm{~d} s \\
\quad-\int_{0}^{+\infty} \int_{\mathbb{R}_{+}} \Psi(s, x+\varepsilon) \frac{\partial}{\partial x}[f(s, x)+b(x+\varepsilon)] \mathrm{d} x \mathrm{~d} s \\
=-\int_{0}^{+\infty} \int_{r}^{+\infty} \frac{\partial p_{D}}{\partial y}(r, s, 0, \varepsilon) \frac{\partial f}{\partial x}(s, 0) \mathrm{d} s \mathrm{~d} V_{r}
\end{aligned}
$$

and

$$
\int_{0}^{+\infty} \int_{\mathbb{R}_{+}} \Gamma(s, x+\varepsilon)\left(\frac{\partial f}{\partial s}(s, x)+\frac{\partial^{2} f}{\partial x^{2}}(s, x)\right) \mathrm{d} x \mathrm{~d} s
$$




$$
\begin{aligned}
& -\int_{0}^{+\infty} \int_{\mathbb{R}_{+}} \Gamma(s, x+\varepsilon) \frac{\partial}{\partial x}[b(x+\varepsilon) f(s, x)] \mathrm{d} x \mathrm{~d} s \\
= & \int_{0}^{\infty} \Gamma(s, \varepsilon) \frac{\partial f}{\partial x}(s, 0) \mathrm{d} s+\int_{0}^{+\infty} \int_{\mathbb{R}_{+}} F(r, x+\varepsilon, u(r, x+\varepsilon)) f(r, x) \mathrm{d} x \mathrm{~d} W_{r} .
\end{aligned}
$$

From the above identities we have that

$$
\begin{aligned}
\int_{0}^{+\infty} \int_{\mathbb{R}_{+}} u(s, x+\varepsilon)\left[\frac{\partial^{2} f}{\partial x^{2}}(s, x)+\frac{\partial f}{\partial s}(s, x)\right] \mathrm{d} x \mathrm{~d} s \\
\quad-\int_{0}^{+\infty} \int_{\mathbb{R}_{+}} \frac{\partial}{\partial x}(b(x+\varepsilon) f(s, x)) u(s, x+\varepsilon) \mathrm{d} x \mathrm{~d} s \\
+\int_{0}^{+\infty} \int_{\mathbb{R}_{+}} F(r, y+\varepsilon, u(r, y+\varepsilon)) f(r, y) \mathrm{d} y \mathrm{~d} W_{r} \\
\quad+\int_{0}^{+\infty} \int_{r}^{\infty} \frac{\partial p_{D}}{\partial y}(r, s, 0, \varepsilon) \frac{\partial f}{\partial x}(s, 0) \mathrm{d} s \mathrm{~d} V_{r}+\int_{0}^{+\infty} \Gamma(s, \varepsilon) \frac{\partial f}{\partial x}(s, 0) \mathrm{d} s .
\end{aligned}
$$

The first term in the right hand side is studied in Lemma 9, where we prove that it converges a.s. to $-\int_{0}^{\infty} \frac{\partial f}{\partial x}(s, 0) \mathrm{d} V_{s}$. Using the same arguments as in Lemma 13 we can show that the function

$$
\int_{0}^{\infty} \Gamma(s, \cdot) \frac{\partial f}{\partial x}(s, 0) \mathrm{d} s
$$

is a.s. continuous on $[0, \infty)$, so that the last integral a.s. converges to zero. This concludes the proof of existence.

Finally, we consider the uniqueness problem. Consider a solution $\tilde{u}(t, x)$, which exists for the first part of the theorem. We introduce the process

$$
v(t, x)=\tilde{u}(t, x)-\int_{0}^{t} \int_{\mathbb{R}_{+}} p_{D}(s, t, y, x) F(s, y, \tilde{u}(s, y)) \mathrm{d} y \mathrm{~d} W_{s}
$$

it is easy to verify that $v(t, x)$ is a weak solution, in the sense of Definition 2, of Eq. (1.1) with $F \equiv 0$. By well known results, we have that $v(t, x)$ is unique and

$$
v(t, x)=\int_{0}^{t} \frac{\partial p_{D}}{\partial x}(s, t, 0, x) \mathrm{d} V_{s}
$$

which implies that $\tilde{u}(t, x)$ satisfies the evolution equation (1.2) and, hence, it is unique. 
In order to complete the proof of the theorem, it is necessary to prove the claims in (5.1) and (5.2). This results from some lengthy computations based on Kolmogorov's equation and integration by parts.

Claim (5.1) is proved by an application of the evolution equation (2.8) in the definition of $\Psi(t, x)$

$$
\begin{aligned}
& \int_{0}^{+\infty} \int_{\mathbb{R}_{+}} \Psi(s, x+\varepsilon) \frac{\partial f}{\partial s}(s, x) \mathrm{d} x \mathrm{~d} s \\
& =\int_{0}^{+\infty} \int_{\mathbb{R}_{+}} \int_{0}^{s} \frac{\partial p_{D}}{\partial y}(r, s, 0, x+\varepsilon) \frac{\partial f}{\partial s}(s, x) \mathrm{d} V_{r} \mathrm{~d} x \mathrm{~d} s \\
& =\int_{0}^{+\infty} \int_{\mathbb{R}_{+}} \int_{0}^{s} \frac{\partial q_{D}}{\partial y}(r, s, 0, x+\varepsilon) \frac{\partial f}{\partial s}(s, x) \mathrm{d} V_{r} \mathrm{~d} x \mathrm{~d} s \\
& \quad+\int_{0}^{+\infty} \int_{\mathbb{R}_{+}}^{s} \int_{0}^{s} \int_{r} b(x+\varepsilon) q_{D}(\sigma, s, z, x+\varepsilon) \frac{\partial^{2} p_{D}}{\partial z \partial y}(r, \sigma, 0, z) \\
& \quad \times \frac{\partial f}{\partial s}(s, x) \mathrm{d} z \mathrm{~d} W_{\sigma} \mathrm{d} V_{r} \mathrm{~d} x \mathrm{~d} s .
\end{aligned}
$$

By repeated applications of Fubini's theorem and integration by parts formula we get

$$
\begin{aligned}
& \int_{0}^{+\infty} \int_{\mathbb{R}_{+}} \Psi(s, x+\varepsilon) \frac{\partial f}{\partial s}(s, x) \mathrm{d} x \mathrm{~d} s \\
& =\int_{0}^{+\infty} \int_{r}^{+\infty} \frac{\partial q_{D}}{\partial y}(r, s, 0, \varepsilon) \frac{\partial f}{\partial x}(s, 0) \mathrm{d} s \mathrm{~d} V_{r} \\
& \quad+\int_{\mathbb{R}_{+}} \int_{0}^{+\infty} \int_{r}^{+\infty} \frac{\partial q_{D}}{\partial y}(r, s, 0, x+\varepsilon) \frac{\partial^{2} f}{\partial x^{2}}(s, x) \mathrm{d} s \mathrm{~d} V_{r} \mathrm{~d} x \\
& \quad+\int_{\mathbb{R}_{+}} \int_{0}^{+\infty} \Psi(\sigma, x+\varepsilon) \frac{\partial}{\partial x}(f(\sigma, x) b(x+\varepsilon)) \mathrm{d} W_{\sigma} \mathrm{d} x \\
& \quad-\int_{\mathbb{R}_{+}} \int_{0}^{+\infty} \int_{r}^{+\infty} \int_{\sigma}^{+\infty} b(\varepsilon) q_{D}(\sigma, s, z, \varepsilon) \frac{\partial^{2} p_{D}}{\partial y \partial z}(r, \sigma, 0, z) \frac{\partial f}{\partial x}(s, 0) \mathrm{d} s \mathrm{~d} W_{\sigma} \mathrm{d} V_{r} \mathrm{~d} z \\
& \quad-\int_{\mathbb{R}_{+}} \int_{\mathbb{R}_{+}}^{+\infty} \int_{0}^{+\infty} \int_{r}^{+\infty} \int_{\sigma}^{+\infty} b(x+\varepsilon) q_{D}(\sigma, s, z, x+\varepsilon) \frac{\partial^{2} p_{D}}{\partial y \partial z}(r, \sigma, 0, z) \\
& \quad \times \frac{\partial^{2} f}{\partial x^{2}}(s, x) \mathrm{d} s \mathrm{~d} W_{\sigma} \mathrm{d} V_{r} \mathrm{~d} z \mathrm{~d} x
\end{aligned}
$$

which is equivalent to (5.1). 
In order to control (5.2), we apply Kolmogorov's equation (2.7):

$$
\begin{aligned}
& \int_{0}^{+\infty} \int_{\mathbb{R}_{+}} \Gamma(s, x+\varepsilon) \frac{\partial f}{\partial s}(s, x) \mathrm{d} x \mathrm{~d} s \\
& =\int_{0}^{+\infty} \int_{\mathbb{R}_{+}} \int_{0}^{s} F(r, x+\varepsilon, u(r, x+\varepsilon)) \frac{\partial f}{\partial s}(s, x) \mathrm{d} W_{r} \mathrm{~d} x \mathrm{~d} s \\
& \quad+\int_{0}^{+\infty} \int_{\mathbb{R}_{+}} \int_{0}^{s} \int_{r}^{s} \int_{\mathbb{R}_{+}} \frac{\partial f}{\partial s}(s, x) \frac{\partial^{2}}{\partial x^{2}} p_{D}(r, \sigma, y, x+\varepsilon) \\
& \quad \times F(r, y, u(r, y)) \mathrm{d} y \mathrm{~d} \sigma \mathrm{d} W_{r} \mathrm{~d} x \mathrm{~d} s \\
& \quad+\int_{0}^{+\infty} \int_{\mathbb{R}_{+}}^{s} \int_{0}^{s} \int_{r} \int_{\mathbb{R}_{+}} \frac{\partial f}{\partial s}(s, x) b(x+\varepsilon) \frac{\partial}{\partial x} p_{D}(r, \sigma, y, x+\varepsilon) \\
& \quad \times F(r, y, u(r, y)) \mathrm{d} y \mathrm{~d} W_{\sigma} \mathrm{d} W_{r} \mathrm{~d} x \mathrm{~d} s .
\end{aligned}
$$

A little effort, using the same ideas as in the lines leading to (5.1), shows that this quantity actually matches (5.2).

\section{REFERENCES}

[1] Alòs E., Nualart D., An extension of Itô’s formula for anticipating processes, J. Theoret. Probab. 11 (2) (1998) 493-514.

[2] Alòs E., Nualart D., Viens F., Stochastic heat equation with white-noise drift, Ann. Inst. H. Poincaré (Probab. Statist.) 36 (2) (2000) 181-218.

[3] Bonaccorsi S., Stochastic variation of constants formula for infinite dimensional equations, Stoch. Anal. Appl. 17 (4) (1999) 509-528.

[4] Da Prato G., Zabczyk J., Evolution equations with white-noise boundary conditions, Stochastics Rep. 42 (3-4) (1993) 167-182.

[5] Freidlin M.I., Wentzell A., Reaction-diffusion equations with randomly-perturbed boundary conditions, Ann. Probab. 20 (1992) 963-986.

[6] Garsia A., Rodemish E., Rumsey H., A real variable lemma and the continuity of paths of some Gaussian processes, Indiana Univ. Math. J. 20 (1970) 565-578.

[7] Hu Y., Nualart D., Continuity of some anticipating integral processes, Statist. Probab. Lett. 37 (1998) 203-211.

[8] Kifer Y., Kunita H., Random positive semigroups and their random infinitesimal generators, in: Davies I.M., Truman A., Elworthy K.D. (Eds.), Stochastic Analysis and Applications, World Scientific, 1996, pp. 270-285.

[9] Kunita H., Stochastic Flows and Stochastic Differential Equations, Cambridge University Press, 1990.

[10] Kunita H., Generalized solutions of a stochastic partial differential equation, J. Theoret. Probab. 7 (1994) 279-308.

[11] Maslowski B., Stability of semilinear equations with boundary and pointwise noise, Ann. Scuola Norm. Sup. Pisa Cl. Sci. (4) 22 (1) (1995) 55-93.

[12] Nualart D., The Malliavin Calculus and Related Topics, Springer-Verlag, New York, 1995. 
[13] Nualart D., Pardoux E., Stochastic calculus with anticipating integrands, Probab. Theory Related Fields 78 (1988) 535-581.

[14] Nualart D., Viens F., Evolution equation of a stochastic semigroup with white-noise drift, Ann. Probab. 28 (1) (2000) 36-73.

[15] Skorohod A.V., On a generalization of a stochastic integral, Theory Probab. Appl. 20 (1975) 219-233.

[16] Sowers R.B., Multidimensional reaction-diffusion equations with white noise boundary perturbations, Ann. Probab. 22 (4) (1994) 2071-2121.

[17] Zakai M., Some moment inequalities for stochastic integrals and for solutions of stochastic differential equations, Israel J. Math. 5 (1967) 170-176. 